\title{
Surface Chemistry Provoked Strategy to Develop Sustainable Latent Fingerprints Visualization and Multiple Anti-Counterfeiting Trajectory: Aggregation- Induced Emission Mechanism Based Active Conjugated Imidazole Luminogens
}

\author{
M. K. Ravindra
}

Kuvempu University

\section{Giriyapura Prabhukumara Darshan}

FMPS, Ramaiah University of Applied Sciences

D. R. Lavanya

Tumkur University

K. M. Mahadevan

Kuvempu University

\section{H. B. Premkumar}

FMPS, Ramaiah University of Applied Sciences

S. C. Sharma

National Assessment and Accreditation Council

H. Adarsha

Jain University

H. Nagabhushana ( $\nabla$ bhushanvlc@gmail.com )

Tumkur University

\section{Research Article}

Keywords: Conjugated luminogens, Aggregation-induced emission, "on-on" fluorescence, Latent fingerprints, Anti-counterfeiting

Posted Date: May 10th, 2021

DOI: https://doi.org/10.21203/rs.3.rs-441794/v1

License: (c) (1) This work is licensed under a Creative Commons Attribution 4.0 International License. Read Full License 
Version of Record: A version of this preprint was published at Scientific Reports on August 18th, 2021. See the published version at https://doi.org/10.1038/s41598-021-96011-5. 


\section{Abstract}

Aggregation-induced emission based organic heterocyclic luminogens bearing conjugated electronic structure have paid much attention due to its excellent fluorescence in aggregation state. In this communication, novel conjugated blue light emitting imidazole molecule is synthesized by one pot multicomponent reaction route is reported for the first time. The prepared molecule exhibits a strong fluorescence in aggregation state have gain much attention owing to their unique properties, namely simple synthesis, high purity, inexpensive, eco-friendly, large scale production, high photostability, etc. By considering these advantages of the prepared luminogen, a new fluorescence based platform has been setup for in-situ visualization of latent fingerprints and its preservation by spray method followed by Poly(vinyl alcohol) masking. A clear and well defined fluorescence fingerprint images on variety of surfaces by revealing level 1-3 ridge characteristics upon ultraviolet $365 \mathrm{~nm}$ light are noticed. The dual nature of binding specificity as well as excellent fluorescence properties permits the visualization of latent fingerprints for longer durations (up to 365 days) with superior contrast, sensitivity, efficiency, selectivity and negligible background hindrance. We further fabricated unclonable invisible security ink and make it highly suitable for various printing modes on valuable goods for protection against forging. The developed labels are displaying uniform distribution of ink and exceptional stability under various atmospheric environments. The development of long preservative forensic information as well as invisible ink opens new avenue in advanced forensic and data security applications.

\section{Introduction}

Fingerprints (FPs) are considered to be personal identity cards as well as information banks owing to their unique permanent features ${ }^{1,2}$. As a result, they can be utilized in unlock tools, biometric identification and similar authentication purposes. Most of the detectable FPs during crime investigation are latent and consequently their visualization has created new avenue in various fields, namely medical diagnostics, forensic investigation and health assessment ${ }^{3-5}$. Till date, numerous chemical as well as physical methods were attempted for visualization of latent fingerprints (LFPs). However, most of the traditional techniques suffer from severe drawbacks, namely low sensitivity, contrast, selectivity, sturdy background hindrance as well as high toxicity ${ }^{6-10}$. Among them, most commonly used powder dusting technique damages the FPs ridge characteristics at the time of staining and also dust is inevitably dangerous to the developers. Moreover, the chemicals used in those techniques can create a problem to the eyes, skin, DNA or mucous membranes ${ }^{11-15}$. The spraying technique is versatile for developing LFPs on various simple and complex surfaces and exhibit plentiful advantages, including simplistic, rapid, superior sensitivity and selectivity, easy-to-store, cost-effective, as well as large-area operable, which is considered to be utmost favourable technique for visualization of LFPs ${ }^{16}$. In image processing, digital data can be forged or easily ruined. As a result, protection of storage along with effortless retrieval technique is highly essential. It is the matter of high concern once it involves the FPs of extremely wanted criminals ${ }^{17}$. The digitally stored FPs can be effortlessly manipulated by many ways. Several methods have been attempted to secure the digitally stored FPs. For instance, Draper et al. ${ }^{18}$ proposed a simple 
method to encode fingerprint biometrics securely for long storage, but fails in system security as well as the detailed authentication. In addition, many research communities are seriously attempted on cryptographic aspects of the problem ${ }^{19-21}$. Aforementioned techniques, encoded FPs have also stored digitally, which can be subsequently manipulated ${ }^{22}$. Hence, physically developed FPs which can be preserved longer duration is highly essential for crime investigations.

In recent years, counterfeiting of security documents, namely currency, certificates, passport, pharmaceutical products have significantly imbalances the economic condition of the world ${ }^{23-25}$. In addition, easy accessibility of anti-counterfeiting (AC) inks and its validation for the counterfeiters are the key anxieties in this area. Hence, progress of the AC and authentication methods having multilevel securities is a key aspect to combat counterfeit ${ }^{26,27}$. Till date, stimuli-chromic as well as photoluminescent materials, namely quantum dots, carbon dots, polymer nanoparticles, organic and inorganic metal complexes, supramolecular structures, polymer dots, up and down-converting nanomaterials, etc. are most reliable materials for fabrication of complex AC inks ${ }^{28-32}$. For instance, the carbon dots generally display broad emission spectra and frequently cause overlapping of spectroscopic profiles $^{33}$. Moreover, semiconductor quantum dots prepared using hazardous raw materials can emit narrow emission peaks ${ }^{34}$. In addition, synthesis of metal oxide frameworks is found to be low yield and display excellent luminescent properties with superior compatibility. Hence, fabrication of novel materials to overcome from the existing limitations is still a challenging task for material scientists.

Aggregation-induced emission (AIE) based organic fluorescent materials finds numerous advantages owing to its intensive fluorescence, excellent contrast, simple functionalization procedure as well as intrinsic AIE properties ${ }^{35,36}$. In addition, these materials can also be utilized in imaging and sensing agents. Most of the fluorescent materials suffers severe self-quenching in its aggregation state. From past decades, AIE based materials were utilized to visualize LFPs and AC ink, due to their less toxicity, easy development procedure, superior resolution, etc. ${ }^{37,38}$. On the other hand, some of the AIE based materials also have drawbacks in real-time applications, including large quantity of material required, chance of disturbing the FPs ridge characteristics at the time of staining process, inhalation of the dust particles may be harmful to the developers and typically suitable for smooth substrates ${ }^{39,40}$. Table S1 shows the reported AIE based materials for visualization of LFPs and AC applications ${ }^{41-49}$. Therefore, exploring the new AIE based fluorescent materials are in urgent need to avoid these limitations.

To address the above issues, it is essential to fabricate alternate probes for LFPs visualization as well as AC applications by showcase the following characteristics: (i) simple fabrication and development procedure, (ii) non-toxicity, (iii) avoiding aggregation induced quenching, (iv) intensive "on - on" fluorescence mode with high photostability, (v) applicable for porous/semi-porous/non-porous surfaces and (vi) long storage ability of the developed FPs. Herein, we have fabricated an 2-[1-(9H-fluoren)-4,5diphenyl-1H-imidazol-zyl]phenol (FDIP) molecule with intense blue emission. The FDIP molecule exhibit exceptional ability on visualization of LFPs with fluorescence "on - on" mode by spraying with an optimized FDIP solution under ultraviolet (UV) $365 \mathrm{~nm}$ light illumination. The detailed investigation of 
LFPs development dynamics, effect of dye concentration, stability of the solution, aging of LFPs, prolonged FPs storage and effect of various environmental conditions and effect of substrates generality is clearly studied. In addition, level 3 details of developed FPs films, namely sweat pores, shapes of ridge end, distribution of pores are analysed. Furthermore, we have also developed AC labels by following simple stamping, writing pen, ink-free relief and intaglio printing techniques that relies on UV $365 \mathrm{~nm}$ light to encrypt input AC details on the various substrates. Further, the encrypted input labels are highly stable under different environmental conditions, which signify the favourable applications in the current AC area.

\section{Synthesis Of 2-[1-(-fluoren)-4,5-diphenyl-1-imidazol-zyl]phenol (Fdip) Molecule}

The 1,2-diphenylethane-1,2-dione (1 $\mathrm{mmol}, 0.210 \mathrm{~g}), 1$-(2-ydroxyphenyl)ethanone (1 mmol, 0.122g), $9 \mathrm{H}$ fluoren-3-amine $(1 \mathrm{mmol}, 0.182 \mathrm{~g})$, ammonium acetate $(1 \mathrm{mmol}, 0.229 \mathrm{~g})$ were taken in an equimolar concentration and dissolved in glacial acetic acid $(\sim 30 \mathrm{ml})$. The mixture was subsequently ultrasonicated ( 10-20 $\mathrm{min}$ ) and refluxed for $\sim 4-5 \mathrm{~h}$. The chemical reaction was frequently supervised by a thin layer chromatography (TLC) utilizing appropriate ratio of pet ether and ethyl acetate mixture (7:3). Consequently, the resultant mixture was cool down to room temperature (RT) and transferred to ice water $(\sim 250 \mathrm{ml})$. The obtained solution was completely neutralized after the addition of aqueous sodium bicarbonate solution and later on purified through column chromatography by utilizing formerly used solvent chamber ratio to obtained desired compound. Schematic illustration for the synthesis of FDIP molecule was depicted in Fig. 1 (a).

Appearance: White crystalline solid; Yield: $70-75 \%$; mp: $276-278^{\circ} \mathrm{C}$.

Fourier transformed infrared (FT-IR) spectrum $\left(\mathrm{KBr}, \mathrm{cm}^{-1}\right)$

1288.17 (C-0), 1420.89 (Aliphatic C-C), $1601.40(\operatorname{Ar~C}=\mathrm{C}), 1624.44(C=N), 2916.78$ (Aliphatic C-H), 3002.13(Ar C-H), $3427.73(\mathrm{O}-\mathrm{H})$.

${ }^{1} \mathrm{H} \mathrm{NMR}\left(400 \mathrm{MHz}, \mathrm{CDCl}_{3}\right.$ ) : $3.79(\mathrm{~s}, 2 \mathrm{H}), 6.77-6.85(\mathrm{~m}, 2 \mathrm{H}, \mathrm{ArH}), 6.95-7.01(\mathrm{~m}, 2 \mathrm{H}, \mathrm{ArH}), 7.10-7.32(\mathrm{~m}$, $11 \mathrm{H}, \mathrm{ArH}), 7.54-7.66(\mathrm{~m}, 4 \mathrm{H}, \mathrm{ArH}), 8.06-8.14(\mathrm{~m}, 2 \mathrm{H}, \mathrm{ArH})$.

\section{Mass}

$\mathrm{m} / \mathrm{z}=477.18\left(\mathrm{M}^{+1}\right)$.

\section{Characterization}

The Bruker made smart CCD System was utilized for recording the single crystal XRD data utilising Mo$\mathrm{Ka}(\lambda \approx 0.71073 \AA)$ radiation. The SAINT-Plus programme was used to reduce crystal XRD data. Molecular diagrams and mean plane calculations were generated using ORTEP and PARST programs, 
respectively. ${ }^{1} \mathrm{H}$-NMR spectra were recorded using a Jeol-delta with operating frequency of $400 \mathrm{MHz}$ in deuterated chloroform $\left(\mathrm{CDCl}_{3}\right)$ solvent. FTIR spectral data of the prepared samples were recorded using a Nicolet 5700 FT-IR instrument with $\mathrm{KBr}$ pellets. Mass spectrometry (LC-MS) was performed with an 1808036-ABS 4 (0.085) Cm (4:7) mass spectrometer. The Jobin Yvon made Fluorolog-3 spectroflourimeter (Xe lamp, $450 \mathrm{~W}$ ) was used for photoluminescence (PL) studies. High resolution FPs images are captured in a Hitachi (TM-3000) made scanning electron microscope (SEM).

\subsection{Development of long term preservative FPs using prepared FDIP molecule}

The LFPs of healthy donors were used on various porous, semi-porous and non-porous surfaces. The donors are advised to wash their hands with soap and water. Later, the finger was impressed on the surfaces with nominal pressure. The optimized aqueous solution of FDIP molecule (Ethanol: Water/ 30:70) is sprayed on the developed LFPs and air dried. However, the aqueous PVA solution was prepared by dissolving specific amount of PVA in a double distilled water. The specific amount ( $5 \mathrm{ml})$ of PVA solution was wrapped on the developed FPs. Later, the PVA film was peeled slowly and stored in an air tight container to long preservation. The developed FPs on films are photographed in situ using a DSLR Canon EOS 100D camera with $5 \mathrm{~mm}$ focal point (SIGMA MACRO, $50 \mathrm{~mm}, \mathrm{~F} 2.8, \mathrm{EXDG}$ ) under visible and UV $365 \mathrm{~nm}$ light. Figure 1 (b) display the cartoon representation for the development and visualization of long term preservative FPs using optimized FDIP molecule by spraying method followed by Poly(vinyl alcohol) (PVA) wrapping. Abrasion test (physical) was carried out via mounting adhesive tape on the developed FP films and then it peeled off and photographed upon UV light irradiation.

\subsection{Fabrication of anti-counterfeiting ink using prepared FDIP molecule.}

The aqueous solution of FDIP molecule (Ethanol: Water/ 30:70) was filled in fountain and sketch pen as an ink and written on normal paper surface to demonstrate anti-counterfeiting property of the prepared AIE based ink. Furthermore, the anti-counterfeiting patterns were also designed using obtained ink followed by simple stamping method. The encoded patterns are in situ photographed under normal as well as UV $365 \mathrm{~nm}$ light.

\section{Result And Discussions}

\subsection{Chemistry}

The FDIP molecule was prepared via simple acid catalysed to form five membered N-heterocyclic ring. The typical step by step chemical reaction involved in synthesis of FDIP molecule as follows (Fig. 1 (c));

\section{Step-1}

Benzil reacts with ammonium acetate which results in 2-imino-1,2-diphenylethanone. 


\section{Step-2}

A lone pair of electron on nitrogen of 2-imino-1,2-diphenylethanone reacts at imine carbon of Schiff base, which formerly obtained by the condensation of salicylaldehyde and 2-aminoflurene to form 2-( $(9 \mathrm{H}$ fluoren-2-ylamino)(2-hydroxyphenyl)methylimino)-1,2-diphenylethanone.

\section{Step-3}

The 2-((9H-fluoren-2-ylamino)(2-hydroxyphenyl)methylimino)-1,2-diphenylethanone undergoes intramolecular cyclization by rearrangement reaction of lone pair electrons of nitrogen (NH group) at electron deficient carbon of carbonyl group to form an intermediate.

\section{Step-4}

The obtained intermediate undertakes dehydration followed by aromatization to result in final FDIP molecule.

\subsection{AIE feature of FDIP molecule}

Figure 1 (e) depicts photographic images of FDIP molecule dissolved in various solvents, such as dichloromethane (DCM), dimethylformamide (DMF), ethanol as well as tetrahydrofuran (THF) upon normal and UV $365 \mathrm{~nm}$ light illumination. It was evident that, FDIP molecule in ethanol shows highest emission intensity upon UV light irradiation as compared with other solvents. This result was also validated by PL emission spectra of the prepared solutions excited at $\lambda_{\text {Exc }}=365 \mathrm{~nm}$ (Fig. 1 (g)). The spectra exhibit a broad intense peak at $\sim 450 \mathrm{~nm}$, owing to the $\pi \rightarrow \pi^{\star}$ transition as well as aromatic ring rotation. The highest intensity was noticed in FDIP in ethanol solvent, and henceforth this was used for further studies. Further, the photographic images of FDIP molecule dissolved in ethanol/water solutions with various water fractions $\left(f_{w}\right)$ and its corresponding PL emission spectra was shown in Fig. 1 ( $f \& h$ ). As evident from the figure, no significant changes on PL intensity for lower water proportion up to $f_{w}=40$ $\%$. The $P L$ intensity reaches its maximum value at $\mathrm{f}_{\mathrm{w}}=70 \%$ and later gradually diminished. The observed phenomenon is mainly attributed to non-coplanarity in the excited state as well as intramolecular charge transfer state.

\subsection{Spectral data analysis}

FTIR spectrum of the prepared FDIP molecule was depicted in Figure S1. The characteristic bands at 1624 and $1601.40 \mathrm{~cm}^{-1}$ are attributed to $(\mathrm{C}=\mathrm{N})$ in imidazole ring and aromatic $\mathrm{C}=\mathrm{C}$ vibrations in benzene ring, respectively. A sharp absorption bands at $\sim 3002.13$ and $2916.78 \mathrm{~cm}^{-1}$ are corresponding to aromatic and aliphatic $\mathrm{C}-\mathrm{H}$ vibrations. In addition, a broad absorption band at $3427 \mathrm{~cm}^{-1}$, which owing to phenolic $\mathrm{O}-\mathrm{H}$ group. Figure $\mathrm{S} 2$ shows ${ }^{1} \mathrm{H}$ NMR spectrum of the prepared FDIP molecule. As evident from the figure, a singlet at $11.74 \delta$, which confirm the phenolic - $\mathrm{OH}$ group. The noticed large $\delta$ value of proton may be due to intramolecular hydrogen bonding between oxygen and nitrogen. The six multiplet peaks including 21 protons around at 6.38-7.82 $\delta$, which may have assigned to aromatic ring. Further, a 
singlet peak at $3.98 \delta$ have two protons is corresponding to fluorene group. The obtained results clearly evidence the formation of FDIP molecule. The mass spectrum of FDIP molecule shows that the spectral value $\left(\mathrm{m} / \mathrm{z}=477.18\left(\mathrm{M}^{+1}\right)\right)$ was well matched with theoretical value $(\mathrm{m} / \mathrm{z}=476.56)$ (Figure S3). The single crystal FDIP molecule were obtained by solvent evaporation method at RT. Figure 1 (d) shows the ORTEP structure of FDIP molecule. It was clearly evident from the figure that the FDIP molecule have monoclinic system with space group P 21/c and volume of $2460.4(3) \AA^{3}$. The detailed crystal data and structure refinement parameters of FDIP molecule are tabulated in Table S2.

\subsection{Development and Visualization of LFPs films using FDIP solution}

From the past years, the intense research has been attempted to explore the constituents of LFPs, even though study is more complex. Generally, LFPs comprised with numerous constituents instigating from the secretory glands in the dermis, epidermis, intrinsic constituents (metabolites and traces of medications and drugs) as well as extrinsic impurities (moisturisers, dirt, cosmetics, blood, grease, hair care products and food contaminants ${ }^{50}$. Further, both intrinsic and extrinsic components considerably change among individuals (inter-variability) and the same individual from day to day (intra-variability) ${ }^{51}$. Normally, the intrinsic constituents of the LFPs are comprised of water (95-99\%) and organic- inorganic three-dimensional complex emulsions ${ }^{52}$. The eccrine constituents are composed of $\sim 98 \%$ water, besides inorganic and organic compounds. Moreover, up till now 20 more amino acids are quantified in the FPs. In addition, the sebaceous sweat is composed of many organic constituents, among them common are lipids, namely glycerides, cholesterol, fatty acids, squalene, lipid esters as well as sterols. Figure 2 (a) depicts mechanism of LFPs visualization dynamics using FDIP solution. In order to know the main component of the LFPs, a series of solutions were prepared using the main LFPs constituents, namely glucose, glycine, urea, sodium chloride, lactic acid as well as lipids (Fig. 2 (b)). The previously prepared FDIP solutions are soaked on the glass surface and FPs were impressed on it. Later, the FDIP solution was sprayed on the LFPs and visualized under UV $365 \mathrm{~nm}$ light. Well defined clear FPs ridge information revealed on lipids soaked surface than other ones. This may be due to strong interaction between hydrophilic head of the lipids as well as hydrophobic end of FDIP molecule, which results a strong binding among lipids and FDIP molecule (Fig. 2 (a)). The above results clearly reveal that lipids present in LFPs have greater affinity with prepared FDIP molecule, demonstrating the versatility of the FDIP solution for LFPs development. Fluorescence imaging is the prime tool in LFP development due to the high contrast as well as low background interference. In recent years, AIE based materials are considered as more efficient materials to avoid the characteristic aggregation-caused quenching (ACQ) properties of plentiful conventional fluorescent materials. In addition, AIE based molecules are prepared in a binary system solution (usually acetonitrile/water or ethanol/water) exhibit lipophilicity in nature, hence are utilized as an efficient luminescent probes for LFPs visualization. Based on previous reports, development techniques for extraction of LFPs using AIE based molecules are mainly classified as powder dusting and solution method ${ }^{53,54}$. Among them, the solution technique extensively used owing to its rapid, simple operational procedure as well as least materials consumption. Herein, various binary 
mixture concentrations (Ethanol-water fraction) of prepared FDIP molecule for visualization of LFPs films on glass surface upon UV $365 \mathrm{~nm}$ light illumination were performed (Fig. 3 (a-j)). As can be observed from the figures, highly intensive and defined ridge features (type 1-3) were clearly revealed in 7:3 of Ethanol-water fraction. However, visibility of the developed FPs was also retained in other concentrations, but fail to reveal all types of ridge details. Among the studied results, 7:3 of Ethanol-water fraction was found to be superior than other concentrations. Henceforth, this optimized concentration was used for further detailed investigations. The stability of the optimized FDIP solution was examined by extracting FPs films on glass surface with a fresh and aged FDIP solution for 3 months by spraying technique (Fig. $3(\mathrm{k}, \mathrm{l}, \mathrm{n}, \mathrm{o})$ ). Almost indistinguishable clear FP patterns were obtained by using the formerly prepared solutions, and no substantial variations in fluorescence signal contrast. In addition, changes in the emission intensity of the FPs furrows and ridges across the yellow box was studied (Fig. $3(m, p)$ ). Gray scale profiles clearly show the fluorescent signal indicating that the FDIP solution exactly stocked on the ridges, however, no signals on the furrows area owing to not any sweat secretions. These results demonstrate the stability of the optimized FDIP solution even for longer durability.

The FPs composition are mostly inconstant, as substantial variations occur after impression via surface interactions as well as various decomposition and oxidative mechanisms. Normally, FPs compositions can be categorized as (i) initial composition and (ii) the aged composition. These compositions vary via several biological, chemical as well as physical methods ensuing in the aged composition ${ }^{55}$. Hence, much efforts have been required in order to investigate the LFPs mechanisms over various periods. This was broadly categorized into two prospective fields; (i) the LFPs development techniques and (ii) the enhancement of aged fingerprints. Better understanding of LFPs constituents offers for augmented knowledge of the mechanisms and chemical kinetics that happen between development reagents and their specific FP constituents. This open up new area of research for advancement in materials and developmental techniques results in extraordinary level of efficiency for both fresh as well as aged FPs. Furthermore, the conventional reagents, namely Nile red and oil red $\mathrm{O}$ are unsuccessful for development of LFPs aged up to 4 weeks, owing to loss of moisture, dispersion of water-soluble components as well as fragile constituent's degradation with respect to time ${ }^{56}$. In the present work, we explored the effectiveness of the both development technique and chemical reagent for the visualization of aged LFPs. The developed FPs films using FDIP solution were stored for 1, 7, 30 and 360 days on glass plate (Fig. 4 (a-d)). As can be seen from the figure, upon UV $365 \mathrm{~nm}$ light nearly identical images which comprising all the ridge features were clearly observed. The PVA coating improves the physical adhesiveness of the FDIP molecule on various substrates and particularly results in superior binding of FDIP on PVA to FPs ridges owing to its intrinsic amphiphilicity of PVA. Figure $4\left(a^{1}-d^{1}\right)$ depicts 3 dimensional interactive plots of the corresponding FPs. The obtained result evidently showing uniform distribution of the prepared solution over the FPs ridges. Furthermore, the donor's fingers were impressed on the surface of the glass substrate and subsequently aged for 1, 7, 30 and 90 days (Fig. 4 (e-h)). Later, aged LFPs were visualized by spraying FDIP solution under UV $365 \mathrm{~nm}$ illumination. The achieved result clearly demonstrated that, the visualization sensitivity slowly diminished with prolonged time. However, FPs aged for up to 90 days could be able to reveal characteristic features (including level 1-3). The 
corresponding gray scale profiles of each FP images display distinguishable fluctuations of the gray values between FP ridges and furrows. The above results clearly authenticate the prolonged durability of the developed method and prepared FDIP solution for LFPs visualization. The FPs constituents varies after impression and is affected by donor factors, transfer conditions and environmental factors (light exposure, atmospheric pollution, air circulation, dust, friction, humidity, temperature, etc. $)^{57}$. Most of the FPs on surfaces of the substrates available at the crime spot are undergone severe environmental conditions, which intern affect the detection sensitivity. Among mentioned conditions, light exposure is found to affect much on visibility of FPs. For instance, various findings authenticated that the decomposition of squalene happens quickly upon UV irradiation. Jones et. al. ${ }^{58}$ studied that the variations of lipid components over the period under various environments and also fatty acids were quickly vanished in dark environments. Herein, the photo stability of FDIP solution for visualization of LFPs on glass surface was examined upon prolonged UV $365 \mathrm{~nm}$ illumination up to $\sim 6 \mathrm{~h}$ (Fig. 5 (a-g)). It is apparent that, no obvious fluorescence quenching was noticed. Further, the prepared solution was utilized to visualize the LFPs on the glass surface followed by spray method under UV $365 \mathrm{~nm}$ light. The obtained results clearly showed well defined ridge details, indicating that UV exposure will not influence much on the visualization ability and its corresponding grayscale images are shown in Figure S4. The present results validate the outstanding photo stability of the prepared solution. Generally, the rate of water loss in LFPs was more prominent in temperature and humid environment. The high temperature atmosphere of LFPs results in more degradation of amino acids as compared those at RT. Sampson et. al. ${ }^{59}$ investigated the optimum temperature to develop successful LFPs by utilizing amino acid reagents is found to be $\sim 20-35^{\circ} \mathrm{C}$. The LFPs were treated with heat for longer time period, visualization would be considerably more challenging, apparently owing to amino acid degradation. However, acid salts are significantly resistant for higher temperatures and LFPs are still visible, even after heating at $\sim 70^{\circ} \mathrm{C}$ for $72 \mathrm{~h}$. To validate the sensitivity of the prepared FPs film, a series of experiments were performed by maintaining different temperatures $\left(30,50,100\right.$ and $\left.150^{\circ} \mathrm{C}\right)$ for $30 \mathrm{~min}$ (Fig. $5(\mathrm{~h}-\mathrm{k})$ ). The obtained results clearly indicate that the developed FPs films are highly thermal stable. Besides, variation of the emission intensity between the FP ridges and furrows which exhibit an excellent contrast. It was worth mentioning that the obtained LFPs details clearly withstand even for higher temperatures. The effect of humidity on LFPs development has been attempted under various weather as well as time durations (May, temperature $\sim 45^{\circ} \mathrm{C}$, humidity $47 \%$; September, temperature $\sim 30^{\circ} \mathrm{C}$, humidity $81 \%$; January, temperature $\sim 15^{\circ} \mathrm{C}$, humidity $40 \%$ ) under UV $365 \mathrm{~nm}$ light (Fig. $5(\mathrm{I}-\mathrm{n})$ ). It was presided that no noticeable variations in the clarity of images, which enabling defined level 1-3 minutiae ridge information. To study the versatility of the developed FPs films on different porous (paper with different background, magazine covers), non-porous (glass, stainless steel, plastic and ceramics) and semi-porous surfaces (leather, cardboard, wood and filter paper) under UV $365 \mathrm{~nm}$ irradiation (Fig. 6 (a-I) and corresponding grayscale images are shown in Figure S5). High contrast and resolution FPs images were clearly noticed. The visualized FPs on different surfaces clearly exhibit level 1-3 details with high sensitivity and selectivity. In addition, 3D interactive plots were also studied (Fig. 6 (m-o)). As evident from the figure, prepared FDIP solution staked exactly on the FPs ridges, whereas no signature of fluorescence in furrows regions. The clear fluorescence on the ridges even after drying indicating that the sprayed FDIP solution is not endured 
any outward capillary flow from the center of the liquid towards the edge, showing the suppression of well-known "coffee-ring effect". Furthermore, series of experiments were performed on FPs films after physical abrasion (grayscale images are shown in Figure S6) and photographic images under UV $365 \mathrm{~nm}$ light irradiation (Fig. 7 (a-h)). As evident from the figure, well defined FPs images were photographed even after physical abrasion (8 times). Hence, the present development technique and material are more convenient in storing FPs films for longer duration without any effect in the existence of various physical abrasions. SEM images of the part of the developed FPs films is taken and shown in Fig. 7 (i-n). A clear distribution of FDIP solution over the ridges and reveals level 2 details, which enabling lawful and trustworthy evidence for individualization.

Normally, FPs are distinguished by three levels of information to personal individualization. Level 1 features are not distinctive enough to personal identification, only denotes the shape, center point and triangle points of the FPs. The level 1 features are classified as whorl, loop, arch, etc. The macroscopic Level 2 information, namely bifurcation, lake, cross-over, bridges, island, short ridge, double bifurcation, etc. Level 3 information are the microscopic ridge details of the FP, namely sweat pores, shape of the ridge edge and the width of the ridges ${ }^{60}$. In general, level 1-2 details of FPs are usually available at the crime scene, which is very difficult to individualize the person. Hence, microscopic level 3 information, namely edge shape, width and narrowness of the ridges as well as sweat pores become particularly important ${ }^{61}$. In order to individualize the person, level $2-3$ details are more authoritative due to their unique features. Figure 8 (a) shows the images of level 2-3 information developed on glass surface by using FDIP solution under UV $365 \mathrm{~nm}$ light (The corresponding grayscale images are shown in Figure S7). High magnified fluorescence images of ridge features, namely whorl, ridge end, bifurcation, ridge termination, hook, scar, trifurcation, lake and short ridge are clearly distinguishable. As can be seen from the figure that, the number, position, width of the ridges as well as distribution of characteristic sweat pores on the FPs are effortlessly distinguished. The 3D interactive plot of the portion of the FP clearly show the distribution of sweat pores on the ridges of the FP (yellow markings) (Fig. 8 (b)). Furthermore, fluctuations in the gray value over the FP evidences that the uniform distribution of FDIP solution on the FP ridges (Fig. 8 (c)). Herein, AIE based FDIP solution demonstrates significant competence on long preservative FPs films through "on-on" fluorescence mode by following spraying method upon UV $365 \mathrm{~nm}$ illumination. The present FDIP solution is considered to be excellent fluorescent probe for LFPs development and analysis as compared with previous literature as follows; (i) simple preparation method followed by four-step reactions process, (ii) easy to develop preservative FPs films via simply spraying on the LFPs surfaces, (iii) versatile for many surfaces, namely non-filtrating substrates, (iv) high contrast, superior sensitivity and low background hindrance due to the "on - on" characteristic fluorescent probe and $(\mathrm{v})$ reveal of unprecedented microscopic details of such as sweat pores, shape of the ridge edge and the width of the ridges. Herein, we believe that the "on - on" characteristic fluorescent probe can excel effortlessly in the long preservative FPs development procedure, providing an alternate platform for the forensic scientists.

\subsection{Application to anti-counterfeiting labels}

Page $11 / 31$ 
Exploring real-time applications of the novel materials like FDIP molecule is always significant, due to its exceptional properties, such as high and steady fluorescence emission as well as superior transparency in the visible region, which makes them appropriate candidates for fluorescent ink applications. The characteristic fluorescent "on - on" probe of the optimized FDIP solution made us to attempt anticounterfeiting labels by following simple stamping method. Figure 9 (a) shows schematic illustration of different input anti-counterfeiting patterns developed on paper surface by following stamping method. The photographs of the word "PRINCIPAL INVESTIGATOR IUAC (UGC)", "PRINCIPAL INVESTIGATOR ISRO RESPOND" and "PRINCIPAL INVESTIGATOR Naval Research Board (DRDO)" stamped by using FDIP solution on paper and leather surfaces were shown in Fig. 9 (b). As evident from the figure, stamped labels did not observe on these surfaces under normal light. However, clear images with uniform distribution of the ink was noticed on the encoded patterns upon UV $365 \mathrm{~nm}$ irradiation. In addition, a series of experiments were performed by varying stamping time $(15,30$ and $45 \mathrm{~s})$ on the paper surfaces (Fig. 9 (c)). It was clearly demonstrated that the emission intensity of the encoded patterns upon $365 \mathrm{~nm}$ excitation can be easily engineered by varying the stamping time. Besides, confidential anti-counterfeiting water-mark were developed using optimized FDIP solution on the complex paper surface by stamped method (Fig. 9 (d)). Under normal light, there was no evidence of encoded water-mark on the paper, although highly intensive bright encoded water-mark "PRINCIPAL INVESTIGATOR, ISRO RESPOND" on the complex paper surface was clearly noticed upon UV $365 \mathrm{~nm}$ irradiation. The obtained results clearly demonstrated that the developed stamping method is quite simple and can be used for protecting the confidentiality of the documents. Further, anti-counterfeiting labels are developed via a hero, marker and sketch pen mode upon loading with FDIP solution and used for handwritten of English and Kannada characters on the normal writing paper (Fig. 10 (a)). As can be seen from the figures that the texted information is undoubtedly visible under UV $365 \mathrm{~nm}$ light, however under normal light the clear blank paper was observed. Besides, the uniform distribution and emission intensity of the texted information on the normal paper is still reliable even after lengthened writings and can be retained even after 6 months when stored under RT (Fig. 10 (b)). The photostability of the encoded information using FDIP solution upon UV $365 \mathrm{~nm}$ light irradiation was also studied (Fig. 10 (c)). The developed QR code information was placed at different UV irradiation time period $(0,30,60$ and $120 \mathrm{~min})$ and their corresponding images are photographed. The obtained photographs clearly found that the fluorescence property gradually diminished and became ambiguous above a certain time. This is mainly due to the un-decorated portion exhibit intensive fluorescence upon UV light exposure, although intensity of the decorated QR code reached its maximum value. When the un-decorated portion attains its higher fluorescence intensity like QR code, the difference between the QR code as well as un-decorated portion will not be distinguishable. The obtained results clearly demonstrated that the prepared FDIP solution based counterfeiting labels are used for UV-light sensitive smart labels. Photographic images of encoded information on various surfaces, namely currency, marble, cardboard and leaf under normal light and UV $365 \mathrm{~nm}$ light excitation (Fig. 11 (a-j)). It was evident that the encoded information was clearly decoded under UV light, however, it is invisible under normal light. This indicates that the developed AC ink quite useful for most of the industrial applications to combat counterfeiting of the products. The effect of time period and temperature on the encoded pattern was exclusively studied, as shown in Fig. 11 (k-n). The developed 
patterns on the glassy ceramic tile can withstand its fluorescence up to 30 days at RT. When the patterns are treated with below $90^{\circ} \mathrm{C}$ for 24 hrs showed any drastic alterations of the fluorescent patterns. Normally, the fluorescent patterns are obliterating under different conditions. In the present work, the developed patterns are heat treated at $\sim 100^{\circ} \mathrm{C}$ for 30 min results in complete encoded information is erased. Figure 11 (o-q) depicts the reliability of encoded patterns on the paper surface by treated with water, oil and acetone for $\sim 12 \mathrm{~h}$. The encoded fluorescence patterns are highly stable without much difference, these outstanding advantages offers the prepared FDIP solution based labels to combat counterfeiting in packaging, food and pharmaceutical industries. These results highlighted the potentiality of the material for smart package material for temperature-sensitive goods.

\subsection{Mechanistic study and ink-free printing}

The relief template technique was employed to develop anti-counterfeiting labels by simple procedure as follows; a commercially purchased PVA $(\sim 50 \mathrm{ml})$ and FDIP molecule $(\sim 250 \mathrm{mg})$ were taken in a beaker and irradiated by probe sonicator for $\sim 1 \mathrm{~h}$ by maintained at a frequency of $22 \mathrm{kHz}$ to obtain a clear and transparent solution. The used PVA medium offers excellent dispersion for FDIP molecule without any clusters which resulting superior viscous nature by providing more adhesive property on the various surfaces of the substrates. Later, the resulted mixture was drop-casted onto a surface of the pre-designed shapes, followed by the evaporation of solvents, which results a flexible transparent film. Then the film was peeled slowly from the surface, and pre-designed shapes was exhibited on the film. This developed film was photographed and analysed upon UV $365 \mathrm{~nm}$ irradiation. Figure 12 (a) depicts the schematic representation of simple ink-free relief template method. The relief printing procedure for "lord Ganesha" by using previously prepared mixture as ink-free patterned substrates were shown in Fig. 12 (b). The obtained result clearly displays fluorescent Ganesha pattern on the film, where intensive fluorescence was noticed in the edge areas of the pattern. Figure 12 (c) depicts the process underlying in intaglio printing by using prepared mixture as ink-free patterned substrates. The prepared mixture was poured on the intaglio xylograph, followed by solvent evaporation. Then film was peeled slowly from the surface resulting in a flexible transparent film. The obtained film comprises with well-developed "numericals" profiles upon UV $365 \mathrm{~nm}$ excitation.

\section{Conclusions}

In conclusion, an AlE-based conjugated imidazole molecule is synthesized via one pot multicomponent reaction route. The prepared molecule shows strong blue fluorescence by overwhelming aggregation induced self-quenching. Thanks to the "on-on" property of FDIP molecule, in situ fluorescence visualization of LFPs and invisible security ink was effectively executed. The visualized LFPs images are developed by spraying method followed by PVA masking, which is advantageous for long term preservation with high resolution. The developed FPs reveal level 1-3 ridge features, which are reliable evidences for personal identity. Besides, nanoscopic level-3 ridge information, including ridge end shape, sweat pores distribution and ridge width can be revealed in SEM images. The masked films comprised with FPs acting as a new "information kit" used for protection of data and its effortless retrieval. In 
addition, invisible AC labels are developed by both with and without ink-free techniques. Emission intensity of the encoded patterns upon $365 \mathrm{~nm}$ excitation can be easily engineered by varying the stamping time. The encoded AC labels are easy-reading upon UV $365 \mathrm{~nm}$ light illumination and stable under various conditions. The developed $\mathrm{AC}$ techniques are quite useful to combating counterfeiting in various fields.

\section{Declarations}

Acknowledgment: The authors (G. P. D \& H. B. P) are grateful for sanction of the project DST (SERB), Govt. of India (CRG/2020/006446) and also MS Ramaiah University of Applied Sciences for constant support.

Authors Contribution: The authors M.K.R, G.P.D and H.N are contributed equally to this work. M.K.R and K.M.M designed and synthesized the samples, D.R.L and H.B.P performed the scanning electron microscopy, X-ray diffraction, photoluminescent spectroscopy measurements. M.K.R and D.R.L performed fingerprints experiments. H.A carried out the FTIR and ${ }^{1} \mathrm{H}$ NMR studies. G.P.D wrote the manuscript and analysed the data. S.C.S and $\mathbf{H}$. $\mathbf{N}$ supervision and editing of the manuscript.

Statement of Authors and Informed Consent. The authors confirmed that all experiments (taking fingerprints of a volunteer/individual) were performed in accordance with relevant guidelines and regulations. An explicit informed consent was obtained from the anonymous volunteer providing the fingerprints. The individual explicitly allowed the authors to use the data in the present publication. And also authors confirmed that all human experimental protocols were approved by a Tumkur University institutional committee.

Note: The authors declare no competing financial interest.

\section{References}

1. Jessica N. Rabuck-Gibbons.; Jean M. Lodge.; Anna K. Mapp.; Brandon T. Ruotolo. Collision-Induced Unfolding Reveals Unique Fingerprints for Remote Protein Interaction Sites in the KIX Regulation Domain. Am. Soc. Mass Spectrom.2019, 30, 94-102.

2. Chiara Masellis.; Neelam Khanal.; Michael Z. Kamrath.; David E. Clemmer.; Thomas R. Rizzo. Cryogenic Vibrational Spectroscopy Provides Unique Fingerprints for Glycan Identification. Am. Soc. Mass Spectrom.2017, 28, 2217-2222.

3. Joosub Lee.; Chan Woo Lee.; Jong-Man Kim. A Magnetically Responsive Polydiacetylene Precursor for Latent Fingerprint Analysis. ACS Appl. Mater. Interfaces2016, 8, 6245-6251.

4. Lawrence J. Kaplan. Chemistry and Crime: Investigating Chemistry from a Forensic Science Perspective. ACS Symposium Series2019, 1324, 13-34.

5. Juliana Agudelo.; Lenka Halamkova.; Erica Brunelle.; Roselyn Rodrigues.; Crystal Huynh.; Jan Halamek. Ages at a Crime Scene: Simultaneous Estimation of the Time since Deposition and Age of 
Its Originator. Chem.2016, 88, 6479-6484.

6. Benjamin Figueroa.; Yikai Chen.; Kyla Berry.; Andrew Francis.; Dan Fu. Label-Free Chemical Imaging of Latent Fingerprints with Stimulated Raman Scattering Microscopy. Chem.2017, 89, 4468-4473.

7. Kai Song.; Peng Huang.; Chenglin Yi.; Bo Ning.; Song Hu.; Liming Nie.; Xiaoyuan Chen.; Zhihong Nie. Photoacoustic and Colorimetric Visualization of Latent Fingerprints. ACS Nano2015, 12, 1234412348.

8. Ranjit Singh.; Abhishek Kumar Gupta.; Chullikkattil P. Pradeep. Synthesis of a New Series of Organic Solid-State Near-Infrared Emitters: The Role of Crystal Packing and Weak Intermolecular Interactions and Application in Latent Fingerprint Detection. Growth \& Des.2021, 21, 1062-1076.

9. Shaoxiong Zhang.; Weidong Yin.; Zengming Yang.; Yuan Yang.; Zhao Li.; Shengjun Zhang.; Bo Zhang.; Fenghao Dong.; Jiawei Lv.; Bingyang Han.; Ziqiang Lei.; Hengchang Ma. Functional Copolymers Married with Lanthanide(III) Ions: A Win-Win Pathway to Fabricate Rare Earth Fluorescent Materials with Multiple Applications. ACS Appl. Mater. Interfaces2021, 13, 5539-5550.

10. Menglu Li.; Tian Tian.; Yujing Zeng.; Sha Zhu.; Jianyang Lu.; Jie Yang.; Chao Li.; Yongmei Yin.; Genxi Li. Individual Cloud-Based Fingerprint Operation Platform for Latent Fingerprint Identification Using Perovskite Nanocrystals as Eikonogen. ACS Appl. Mater. Interfaces2020, 12, 13494-13502.

11. Meng Wang.; Ye Zhu.; Chuanbin Mao. Synthesis of NIR-Responsive NaYF 4 : Yb, Er Upconversion Fluorescent Nanoparticles Using an Optimized Solvothermal Method and Their Applications in Enhanced Development of Latent Fingerprints on Various Smooth Substrates. Langmuir2015, 31, 7084-7090.

12. Dhanalakshmi Muniswamy.; Hanumanthappa Nagabhushana.; Basavaraj R. B.; Giriyapura Prabhukumar Darshan.; Daruka Prasad B. Surfactant-Assisted $\mathrm{BaTiO}_{3}: \mathrm{Eu}^{3+} @ \mathrm{SiO}_{2}$ Core-Shell Superstructures Obtained by Ultrasonication Method: Dormant Fingerprint Visualization and Red Component of White Light-Emitting Diode Applications. ACS Sus. Chem. \& Eng.2018, 6, 5214-5226.

13. Di Peng.; Xin Wu.; Xiang Liu.; Mengjun Huang.; Dan Wang.; Renlong Liu. Color-Tunable Binuclear (Eu, Tb) Nanocomposite Powder for the Enhanced Development of Latent Fingerprints Based on Electrostatic Interactions. ACS Appl. Mater. Interfaces2018, 10, 32859-32866.

14. Xiang-Yang Dong.; Xiao-Qing Niu.; Zheng-Yong Zhang.; Ji-Shi Wei.; Huan-Ming Xiong. Red Fluorescent Carbon Dot Powder for Accurate Latent Fingerprint Identification using an Artificial Intelligence Program. ACS Appl. Mater. Interfaces2020, 12, 29549-29555.

15. Meng Wang.; Ming Li.; Aoyang Yu.; Jian Wu.; Chuanbin Mao. Rare Earth Fluorescent Nanomaterials for Enhanced Development of Latent Fingerprints. ACS Appl. Mater. Interfaces2015, 7, 28110-28115.

16. You-Hong Chen.; Shih-Yu Kuo.; Wei-Kai Tsai.; Chi-Shiang Ke.; Chia-Hsien Liao.; Chuan-Pin Chen.; Yeng-Tseng Wang.; Hsiu-Wei Chen.; Yang-Hsiang Chan. Dual Colorimetric and Fluorescent Imaging of Latent Fingerprints on Both Porous and Nonporous Surfaces with Near-Infrared Fluorescent Semiconducting Polymer Dots. Chem.2016, 88, 11616-11623.

17. Roland Menzel. E. Detection of Latent Fingerprintsby Laser-Excited Luminescence. Chem. 1989, 61, 557A-561A. 
18. Draper, S.C.; Khisti, A.; Martinian,E.; Vetro, A.; Yedidia, J. S. "Using distributed source coding to secure fingerprint biometrics, "Proceedings of the IEEE International Conference on Acoustics, Speech and Signal Processing (ICASSP '07), USA, 2007, 2, 129-132.

19. Eachan O. Johnson.; Deborah T. Hung. A Point of Inflection and Reflection on Systems Chemical Biology. ACS Chem. Bio.2019, 14, 2497-2511.

20. Hong Chen.; Rong-liang Ma.; Yun Chen.; Li-Juan Fan. Fluorescence Development of Latent Fingerprint with Conjugated Polymer Nanoparticles in Aqueous Colloidal Solution. ACS Appl. Mater. Interfaces 2017, 9, 4908-4915.

21. Brent Friesen. J. Activities Designed for Fingerprint Dusting and the Chemical Revelation of Latent Fingerprints. Chem. Edu. 2015, 92, 505-508.

22. Ruimin Li.; Yuting Zhang.; Jing Tan.; Jiaxun Wan.; Jia Guo.; Changchun Wang. Dual-Mode Encoded Magnetic Composite Microsphere Based on Fluorescence Reporters and Raman Probes as Covert Tag for Anticounterfeiting Applications. ACS Appl. Mater. Interfaces 2016, 8, 14, 9384-9394.

23. Bai-Heng Wu.; Chao Zhang.; Ning Zheng.; Lian-Wei Wu.; Zhi-Kang Xu.; Ling-Shu Wan. Grain Boundaries of Self-Assembled Porous Polymer Films for Unclonable Anti-Counterfeiting. ACS Appl. Polymer Mat.2019, 1, 47-53.

24. Chen Li.; Xiaoyan Sun.; Yuan Li.; Hailu Liu.; Bibo Long.; Dong Xie.; Junjia Chen.; Ke Wang. Rapid and Green Fabrication of Carbon Dots for Cellular Imaging and Anti-Counterfeiting Applications. ACS Omega2021, 6, 3232-3237.

25. Minzan Zuo.; Weirui Qian.; Tinghan Li.; Xiao-Yu Hu.; Juli Jiang.; Leyong Wang. Full-Color Tunable Fluorescent and Chemiluminescent Supramolecular Nanoparticles for Anti-Counterfeiting Inks. ACS Appl. Mater. Interfaces2018, 10, 39214-39221.

26. Sergii Kalytchuk.; Yu Wang.; Katerina Polǎkova.; Radek Zboril. Carbon Dot Fluorescence-LifetimeEncoded Anti-Counterfeiting. ACS Appl. Mater. Interfaces 2018, 10, 29902-29908.

27. Facundo M. Fernandez.; Michael D. Green.; Paul N. Newton. Prevalence and Detection of Counterfeit Pharmaceuticals: A Mini Review. \& Eng. Chem. Res. 2008, 47, 585-590.

28. Han-Wen Yang.; Ping Xu.; Bo Ding.; Xiu-Guang Wang.; Zheng-Yu Liu.; Hong-Kun Zhao.; Xiao-Jun Zhao.; En-Cui Yang. Isostructural Lanthanide Coordination Polymers with High Photoluminescent Quantum Yields by Effective Ligand Combination: Crystal Structures, Photophysical Characterizations, Biologically Relevant Molecular Sensing, and Anti-Counterfeiting Ink Application. Growth \& Des.2020, 20, 7615-7625.

29. Amin Abdollahi.; Hossein Roghani-Mamaqani.; Bahareh Razavi.; Mehdi Salami-Kalajahi. Photoluminescent and Chromic Nanomaterials for Anticounterfeiting Technologies: Recent Advances and Future Challenges. ACS Nano2020, 14, 14417-14492.

30. Haihu Tan.; Guo Gong.; Shaowen Xie.; Ya Song.; Changfan Zhang.; Na Li.; Dong Zhang.; Lijian Xu.; Jianxiong Xu.; Jie Zheng. Upconversion Nanoparticles@Carbon Dots@Meso-SiO 2 Sandwiched Core-Shell Nanohybrids with Tunable Dual-Mode Luminescence for 3D Anti-Counterfeiting Barcodes. Langmuir2019, 35, 11503-11511. 
31. Diwei Zhang.; Wei Zhou.; Quanlin Liu.; Zhiguo Xia. $\mathrm{CH}_{3} \mathrm{NH}_{3} \mathrm{PbBr}_{3}$ Perovskite Nanocrystals Encapsulated in Lanthanide Metal-Organic Frameworks as a Photoluminescence Converter for AntiCounterfeiting. ACS Appl. Mater. Interfaces2018, 10, 27875-27884.

32. Deepthi N. H.; Giriyapura Prabhukumar Darshan.; Basavaraj R. B.; Daruka Prasad B.; Sharma S. C.; Kavyashree D.; Hanumanthappa Nagabhushana. Nanostructured Stannic Oxides for White Light Emitting Diodes Provides Authentication for Latent Fingerprints Visualization under Diverse Environmental Conditions. ACS Sus. Chem. \& Eng.2019, 7, 578-591.

33. Vasilios Georgakilas.; Jason A. Perman.; Jiri Tucek.; Radek Zboril. Broad Family of Carbon Nanoallotropes: Classification, Chemistry, and Applications of Fullerenes, Carbon Dots, Nanotubes, Graphene, Nanodiamonds, and Combined Superstructures. Rev.2015, 115, 4744-4822.

34. Ilaiyaraja P.; Pavana S. V. Mocherla.; Srinivasan T. K.; Sudakar C. Synthesis of Cu-Deficient and ZnGraded Cu-In-Zn-S Quantum Dots and Hybrid Inorganic-Organic Nanophosphor Composite for White Light Emission. ACS Appl. Mater. Interfaces2016, 8, 12456-12465.

35. Juan Liu.; Yan-Qing Fan.; Shan-Shan Song.; Guan-Fei Gong.; Jiao Wang.; Xiao-Wen Guan.; Hong Yao.; You-Ming Zhang.; Tai-Bao Wei.; Qi Lin. Aggregation-Induced Emission Supramolecular Organic Framework (AIE SOF) Gels Constructed from Supramolecular Polymer Networks Based on Tripodal Pillar[5]arene for Fluorescence Detection and Efficient Removal of Various Analytes. ACS Sus. Chem. \& Eng. 2019, 7, 11999-12007.

36. Farzaneh Rouhani.; Ali Morsali.; Pascal Retailleau. Simple One-Pot Preparation of a Rapid Response AIE Fluorescent Metal-Organic Framework. ACS Appl. Mater. Interfaces2018, 10, 36259-36266.

37. Xue You.; Guanxin Zhang.; Chi Zhan.; Yuancheng Wang.; Deqing Zhang. New Chemo-/Biosensors Based on the Aggregation-Induced Emission Materials and Applications 2016, 2, 93-127.

38. Ju Mei.; Nelson L. C. Leung.; Ryan T. K. Kwok.; Jacky W. Y. Lam.; Ben Zhong Tang. AggregationInduced Emission: Together We Shine, United We Soar! Rev. 2015, 115, 11718-11940.

39. Zeng Xu.; Jiabao Gu.; Xianfeng Qiao.; Anjun Qin.; Ben Zhong Tang.; Dongge Ma. Highly Efficient Deep Blue Aggregation-Induced Emission Organic Molecule: A Promising Multifunctional

Electroluminescence Material for Blue/Green/Orange/Red/White OLEDs with Superior Efficiency and Low Roll-Off. ACS Photonics 2019, 6, 767-778.

40. Pengbo Han.; Chengwei Lin.; Dongge Ma.; Anjun Qin.; Ben Zhong Tang. Violet-Blue Emitters Featuring Aggregation-Enhanced Emission Characteristics for Nondoped OLEDs with CIEy Smaller than 0.046. ACS Appl. Mater. Interfaces 2020, 12, 46366-46372.

41. Xiaodong Jin; Ran Xin,; Shifan Wang,; Wenzhu Yin,; Tongxiang Xu,; Yang Jiang,; Xuran Ji, Luyang Chen,; Jingning Liu. A Tetraphenylethene-based Dye for Latent Fingerprint Analysis. Act. B: Chem. 2017, 17 30079-30084.

42. Raghupathy Suresh; Senthil Kumar Thiyagarajan; Perumal Ramamurthy, An AIE based fluorescent probe for digital lifting of latent fingerprint marks down to minutiae level. Act. B: Chem. 2018, 258, 184-192. 
43. Harminder Singh; Rashmi Sharma; Gaurav Bhargava; Subodh Kumar; Prabhpreet Singh, AIE+ESIPT based red fluorescent aggregates for visualization of latent fingerprints. New J. Chem.2018, 42, 12900-12907.

44. Ya-Long Wang; Chong Li; Hong-Qing Qu; Cheng Fan; Peng-Ju Zhao; Rui Tian; Ming-Qiang Zhu; RealTime Fluorescence In Situ Visualization of Latent Fingerprints Exceeding Level 3 Details Based on Aggregation-Induced Emission. Am. Chem. Soc. 2020, 142, 7497-7505.

45. Akhtar Hussain Malik, Anamika Kaila, Parameswar Krishnan lyer, Development of Well-Preserved, Substrate-Versatile Latent Fingerprints by Aggregation-Induced Enhanced Emission-Active Conjugated Polyelectrolyte. ACS Appl. Mater. Interfaces2017, 9, 37501-37508.

46. Banchhanidhi Prusti,; Manab Chakravarty, Carbazole-Anthranyl $\pi$-Conjugates as Small and Stable Aggregation-Induced Emission-Active Fluorogens: Serving as a Reusable and Efficient Platform for Anticounterfeiting Applications with an Acid Key and Multicolor Ink for a Quill Pen. ACS Omega2019, $42019,4,16963-16971$.

47. Ravindra, M. K.; Mahadevan K. M.; Basavaraj, R. B.; Darshan, G. P.; Sharma, S. C.; Raju, M. S.; Vijayakumar, G. R.; Kiran B. Manjappa, Ding-Yah Yang, Nagabhushana, H. New design of highly sensitive AIE based fluorescent imidazole derivatives: Probing of sweat pores and anti-counterfeiting applications. Sci. \& Eng. C 2019, 101, 564-574.

48. Parvej Alam;; Nelson, L.C.; Leung,; Jing Zhang,; Ryan, T.K.; Kwok;; Jacky, W.Y.; Lam,; Ben Zhong Tang, AIE-based luminescence probes for metal ion detection. Coord. Chem. Rev.2021, 429, 213693.

49. Linru Xu.; Yan Li,; Shuhong Li,; Rongrong Hu,; Anjun Qin;; Ben Zhong Tang,; Bin Su, Enhancing the visualization of latent fingerprints by aggregation induced emission of siloles. RSC Advances, Accepted Manuscript (2021).

50. Ting Zhang.; Chaoyang Wang.; Xiang Ma. Metal-Free Room-Temperature Phosphorescent Systems for Pure White-Light Emission and Latent Fingerprint Visualization. \& Eng. Chem. Res.2019, 58, 77787785.

51. Hyejin Yoon; Krystal, C.; Belmonte; Tom Kasten,; Randall Bateman,; Jungsu Kim, Intra- and Interindividual Variability of microRNA Levels in Human Cerebrospinal Fluid: Critical Implications for Biomarker Discovery. Reports2017, 7, 12720.

52. Praveen Linga.; Nayef Al-Saifi.; Peter Englezos. Comparison of the Luus-Jaakola Optimization and Gauss-Newton Methods for Parameter Estimation in Ordinary Differential Equation Models. \& Eng. Chem. Res.2006, 45, 4716-4725.

53. Lakshmeesha, T. R.; Sateesh, M. K.; Daruka Prasad, B.; Sharma, S. C.; Kavyashree, D.; Chandrasekhar, M.; Nagabhushana, H. Reactivity of Crystalline ZnO Superstructures against Fungi and Bacterial Pathogens: Synthesized Using Nerium oleander Leaf Extract. Growth \& Des.2014, 14, 4068-4079.

54. ; Nagaraju, G.; Nagabhushana, H.; Basavaraj, R. B.; Raghu, G. K.; Suresh, D.; Rajanaika, H.; Sharma, S. C. Green, Nonchemical Route for the Synthesis of ZnO Superstructures, Evaluation of Its Applications toward Photocatalysis, Photoluminescence, and Biosensing. Crys. Growth \& Des.2016, 16, 68286840. 
55. Paige Hinners.; Madison Thomas.; Young Jin Lee. Determining Fingerprint Age with Mass Spectrometry Imaging via Ozonolysis of Triacylglycerols. Chem.2020, 92, 3125-3132.

56. Brent Friesen. Forensic Chemistry: The Revelation of Latent Fingerprints. ACS Appl. Nano Mat. 2019, 2, 4518-4527.

57. Shijie Zhang.; Ronghua Liu.; Qianling Cui.; Yu Yang.; Qian Cao.; Wenqiang Xu.; Lidong Li. Ultrabright Fluorescent Silica Nanoparticles Embedded with Conjugated Oligomers and Their Application in Latent Fingerprint Detection. ACS Appl. Mater. Interfaces2017, 9, 44134-44145.

58. H Jones, N.; Mansour, D.; Stoilovic, M.; Lennard, C.; Roux, C. The influence of polymer type, print donor and age on the quality of fingerprints developed on plastic substrates using vacuum metal deposition, Forensic Sci. Int.2001, 124 (2-3),167-177.

59. Sampson, K.; Sampson, W. Recovery of latent prints from human skin, Forensic Identif.2005, 55 (3), 362-385.

60. Cai-Feng Wang; Rui Cheng,; Wen-Qing Ji,; Kangzhe Ma,; Luting Ling,; Su Chen. Recognition of Latent Fingerprints and Ink-Free Printing Derived from Interfacial Segregation of Carbon Dots. ACS Appl. Mater. Interfaces2018, 10, 39205-39213.

61. Veasna Soum; Sooyoung Park;; Albertus Ivan Brilian;; Yunpyo Kim; Madeline Y. Ryu; Taler Brazell;; John Burpo, F.; Kevin Kit Parker; Oh-Sun Kwon,; Kwanwoo Shin. Inkjet-Printed Carbon Nanotubes for Fabricating a Spoof Fingerprint on Paper. ACS Omega2019, 4, 8626-8631.

\section{Figures}




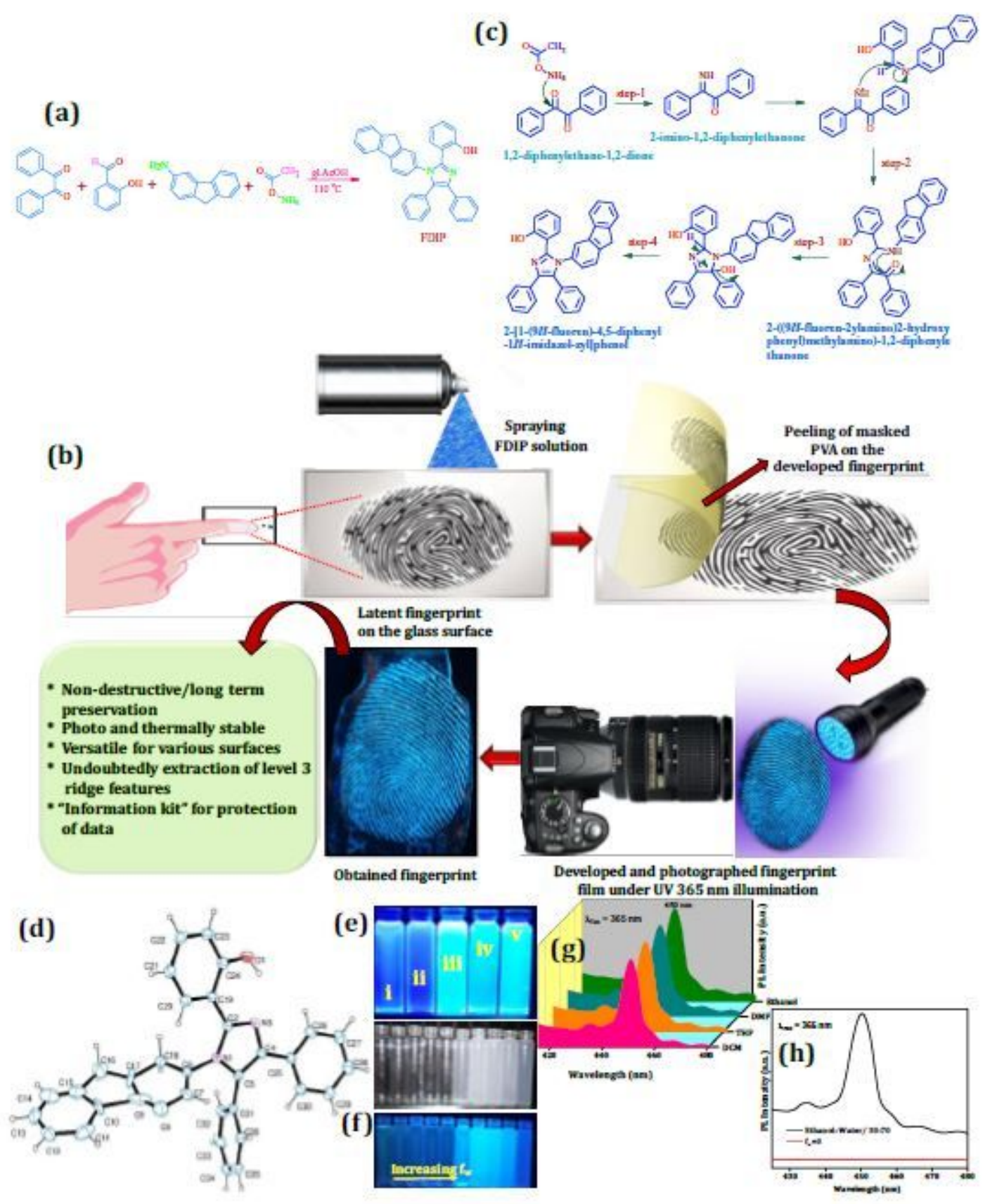

\section{Figure 1}

Schematic illustration for the (a) synthesis of FDIP molecule; (b) Development and visualization of long term preservative FPs using optimized FDIP molecule by spraying method followed by PVA wrapping; (c) Step by step chemical reaction involved in synthesis of FDIP molecule; (d) ORTEP diagram of FDIP molecule. Color code: N-violet, O-red, C-blue; (e) Photographic images of FDIP molecule under UV $365 \mathrm{~nm}$ light in various solvents (i) DCM, (ii) DMF, (iii) ethanol and (iv) THF; (f) AIE images of the FDIP molecule with various fw values in ethanol under normal and $365 \mathrm{~nm}$ light; $(\mathrm{g})$ PL spectra of FDIP molecule in various solvents excited at $365 \mathrm{~nm}$ light; (h) PL spectra of FDIP molecule with $\mathrm{fw}=0$ and ethanol:water/30:70. 


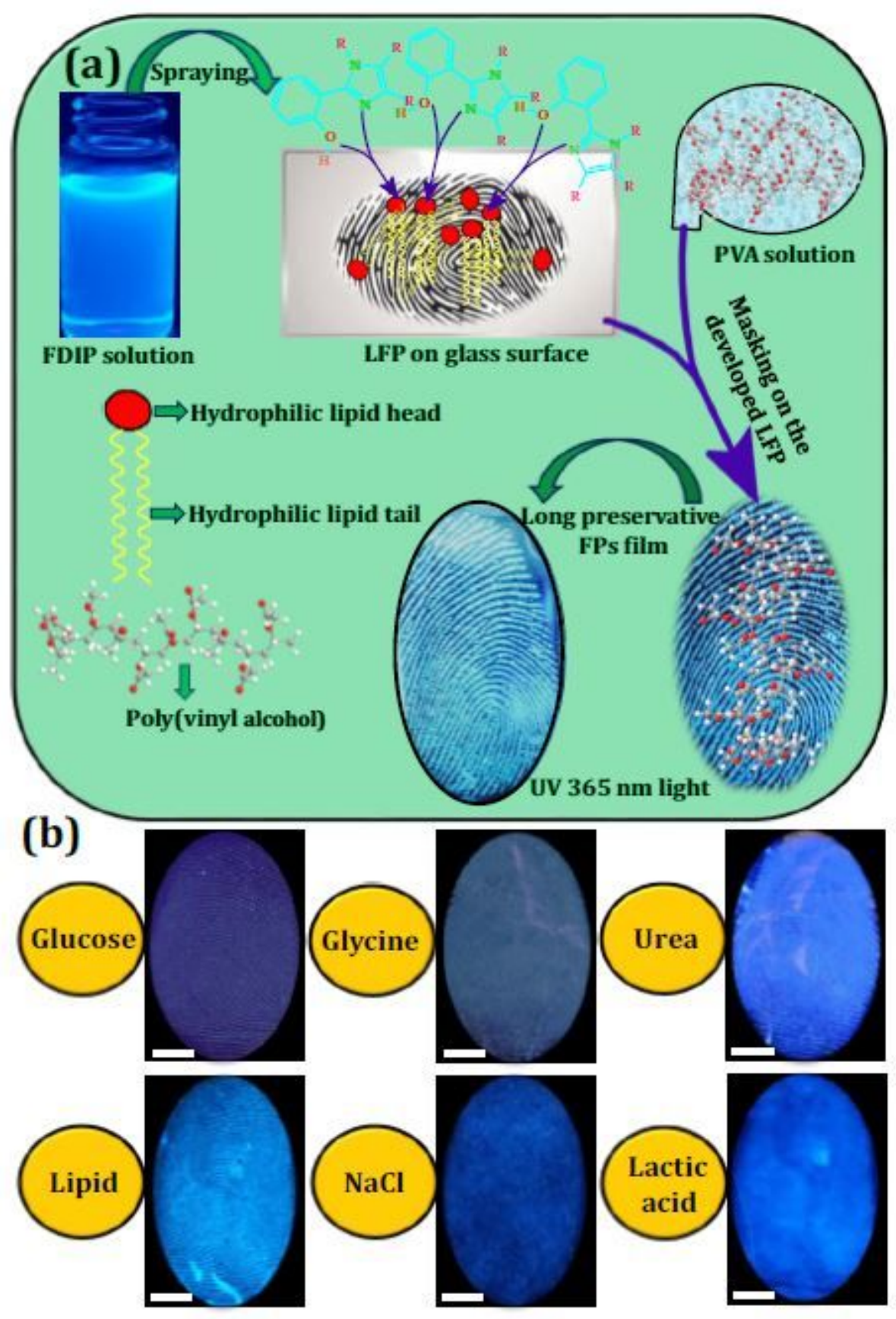

Figure 2

(a) Mechanism of LFPs visualization dynamics using FDIP solution; (b) RGB true-color FPs images developed on the glass surface soaked with glucose, glycine, urea, sodium chloride, lactic acid as well as lipids solutions under $365 \mathrm{~nm}$ light (Scale bar: $5 \mathrm{~mm}$ ). 

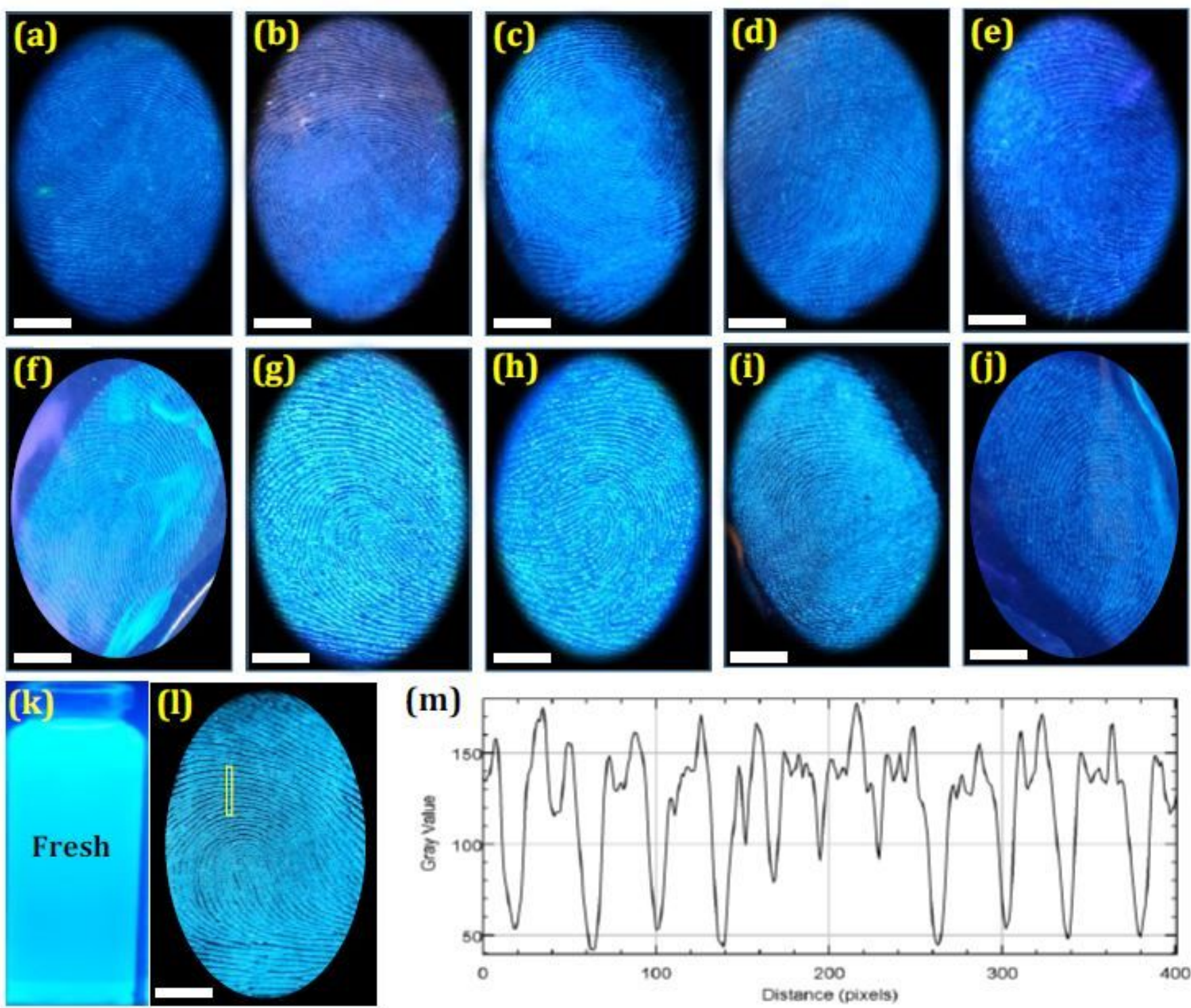

(m)
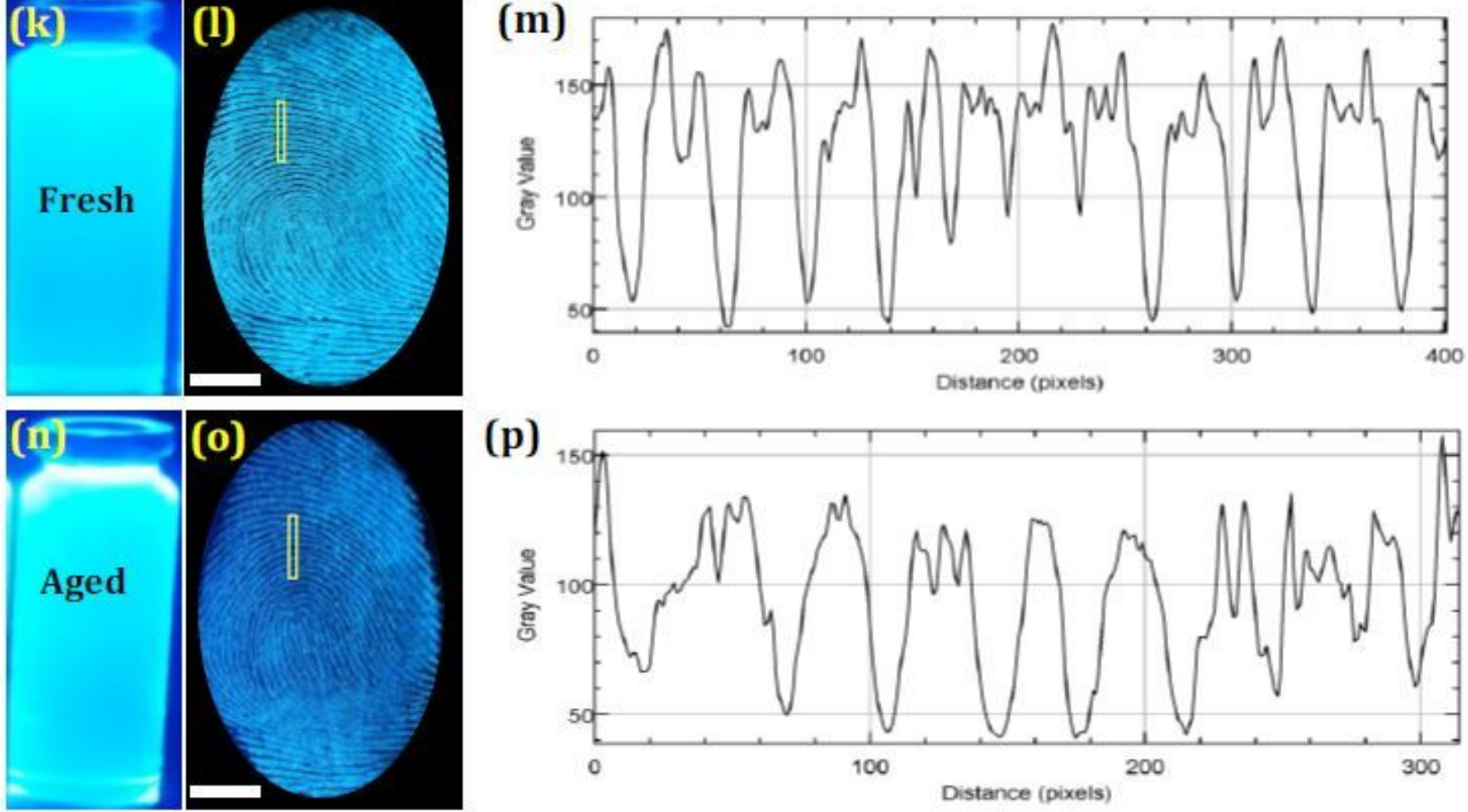

\section{Figure 3}

(a-j) FPs images developed using various binary mixture concentrations (Ethanol-water fraction) of prepared FDIP molecule on glass surface upon UV 365 nm light illumination; ( $k, l, n, 0)$ Fresh and aged FDIP solution after storing for 3 months and used for LFPs development on glass surface; $(m, p)$ Gray scale profiles of the corresponding images of "I and o" (Scale bar: $5 \mathrm{~mm}$ ). 

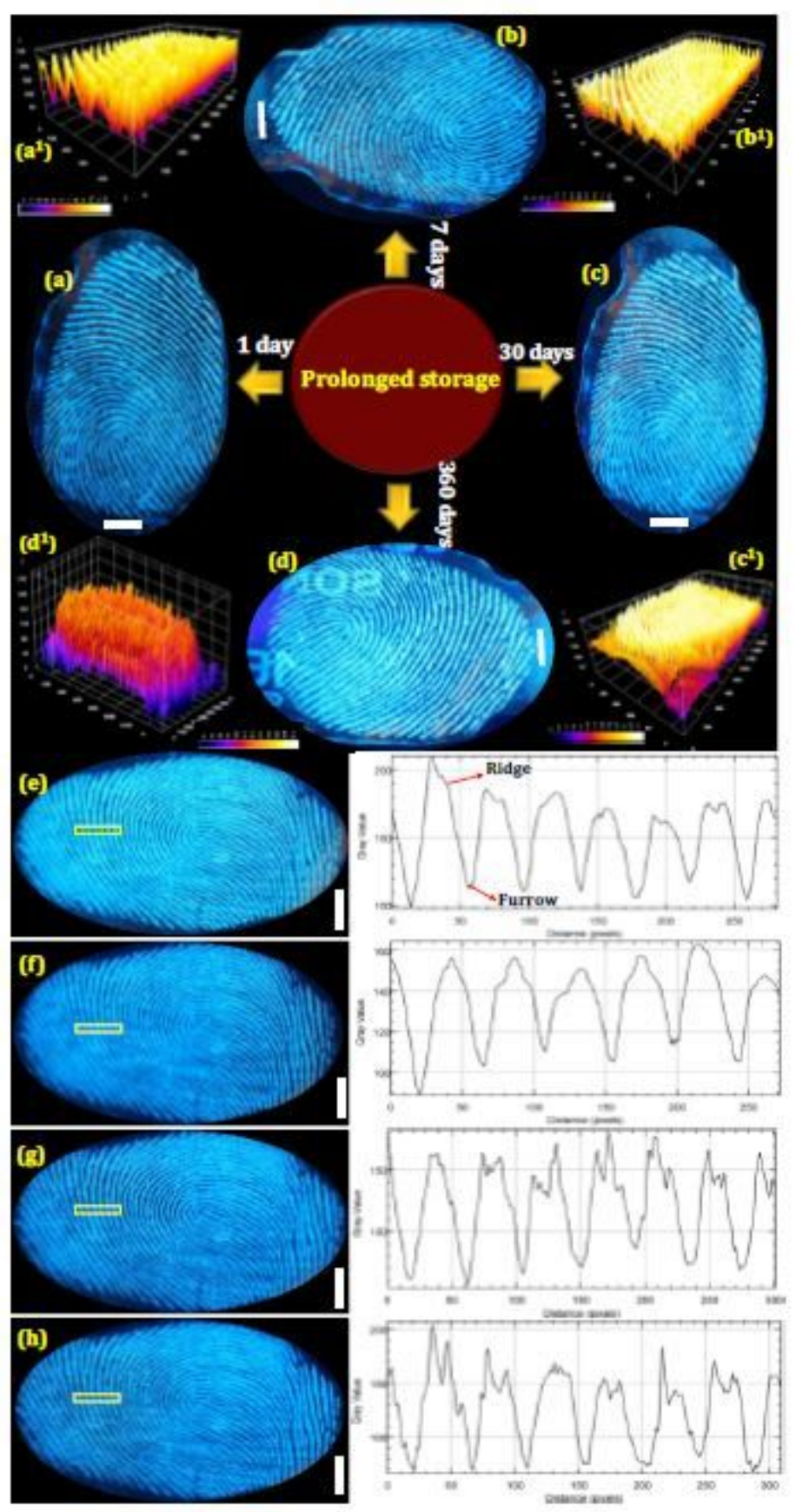

\section{Figure 4}

Fluorescence photographs of FP films developed using FDIP solution on glass surface and stored for (a) 1 day, (b) 7 days, (c) 30 days, (d) 360 days (The FPs are from the same finger); (a1-d1) 3-dimensional interactive plots of the corresponding FP panels "a-d" (showing uniform distribution of the prepared solution over the ridges); (e-h) Fluorescence images of donor fingers impressed on the surface of the glass substrate and subsequently aged for 1, 7, 30 and 90 days. The corresponding gray scale profiles of each images of "e-h", display fluctuations of the gray values between FP ridge and furrow across the yellow line (Scale bar: $5 \mathrm{~mm}$ ). 


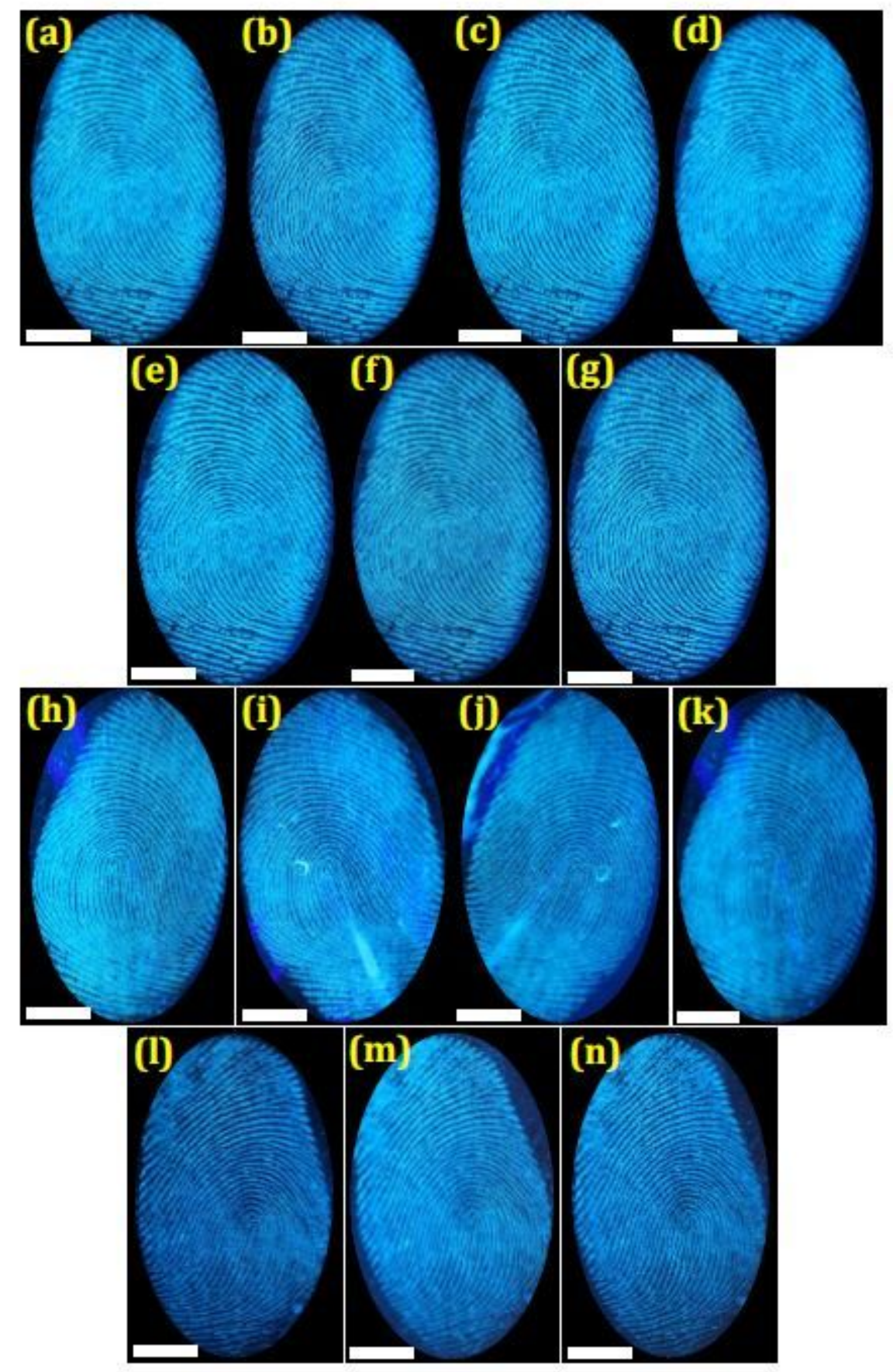

\section{Figure 5}

Stability assessment of FP images developed using FDIP solution on the glass surface; (a-g) developed FPs was examined upon high intensity UV $365 \mathrm{~nm}$ illumination $(2 \mathrm{~mW} / \mathrm{cm} 2) 0$ to $6 \mathrm{hrs}$; (h-k) FPs maintained at different temperatures $\left(30,50,100\right.$ and $\left.150^{\circ} \mathrm{C}\right)$ for $30 \mathrm{~min}$; (I-n) FPs developed under various weather as well as time durations (May, temperature $\sim 45^{\circ} \mathrm{C}$, humidity $47 \%$; September, temperature $\sim 30{ }^{\circ} \mathrm{C}$, humidity $81 \%$; January, temperature $\sim 15^{\circ} \mathrm{C}$, humidity $40 \%$ ) The corresponding grayscale images are shown in Figure S4.(Scale bar: $5 \mathrm{~mm}$ ). 

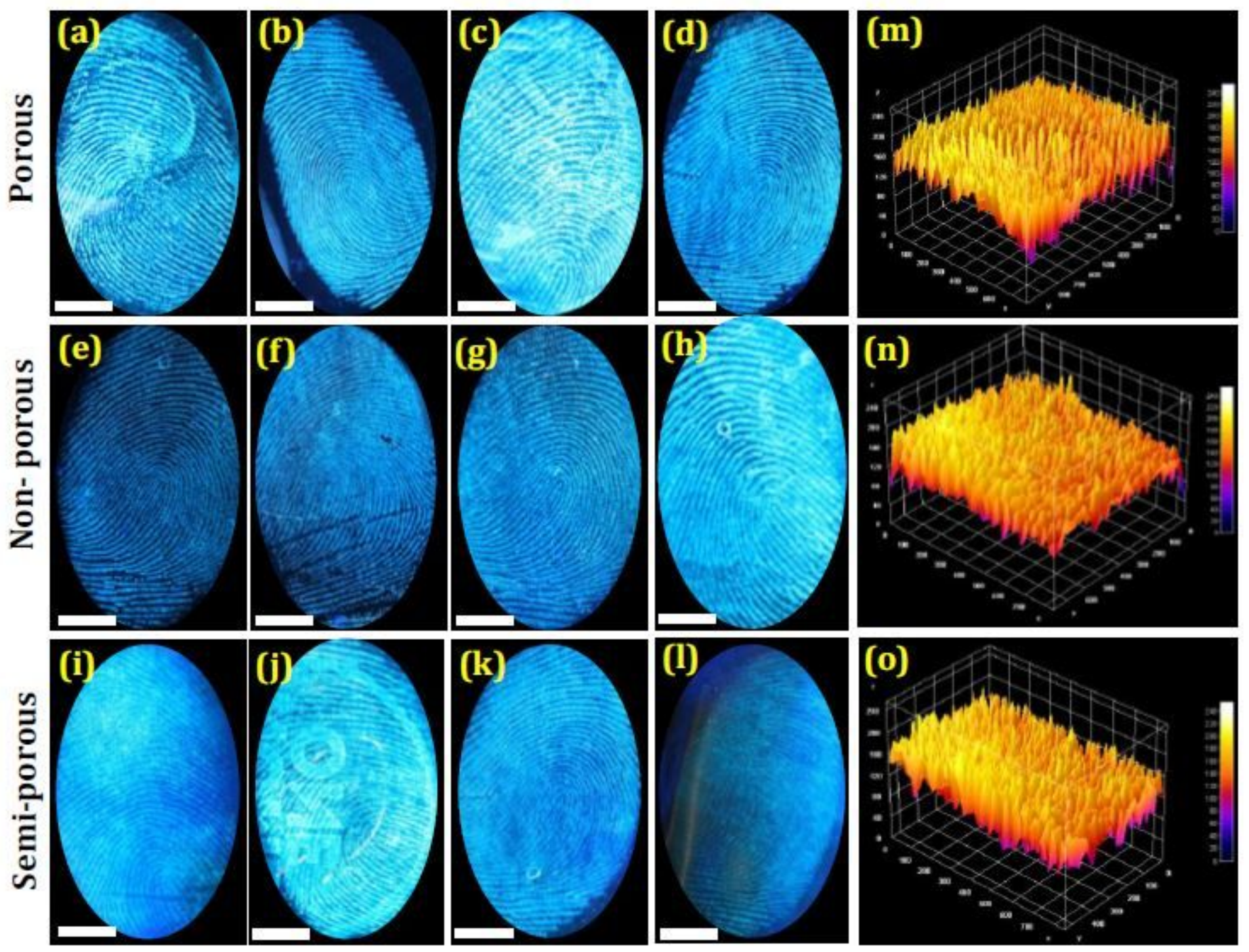

Figure 6

FPs images on different surfaces (a-d) non-porous, (e-h) porous, and (i-l) semi-porous substrates developed by spraying with FDIP solution followed by PVA masking and visualized under UV $365 \mathrm{~nm}$ irradiation. The corresponding grayscale images are shown in Figure S5; (m-o) 3-dimensional interactive plots of the corresponding FP panels "d, e and k", which reveals the uniform distribution of the prepared solution over the ridges (Scale bar: $5 \mathrm{~mm}$ ). 

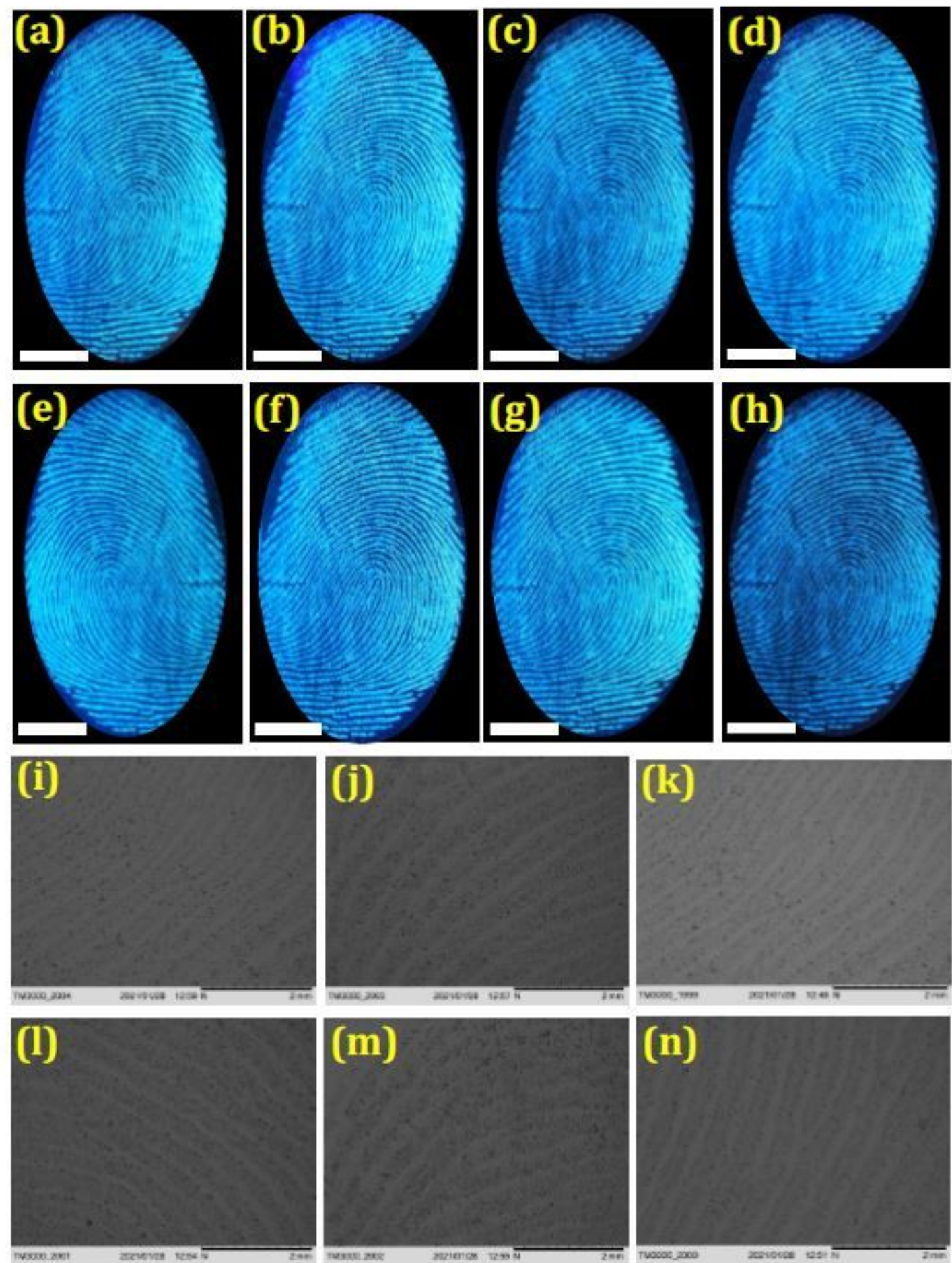

\section{Figure 7}

(a-h) Fluorescent images of FPs after developing with FDIP solution under UV $365 \mathrm{~nm}$ treated with successive physical abrasion. The corresponding grayscale images are shown in Figure S6 (Scale bar: 5 $\mathrm{mm}$ ); (i-n) SEM images of developed FPs reveal various ridge details demonstrating excellent adhesion of FDIP solution with ridges. 

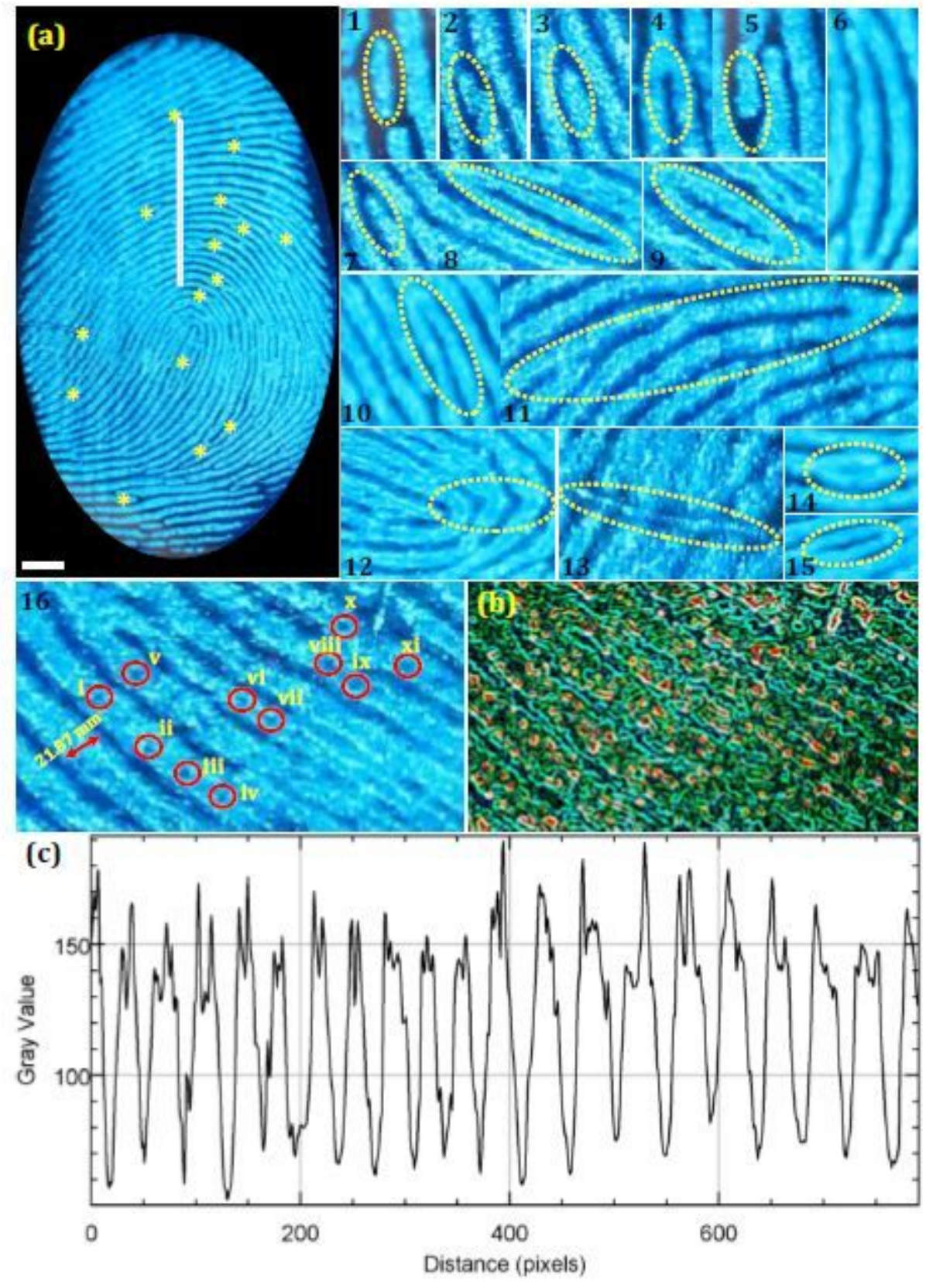

\section{Figure 8}

(a). High resolution FP image along with extracted ridge features including level I, level II and level III (116) on the glass surface visualized by FDIP solution followed by spray method under UV $365 \mathrm{~nm}$ illumination. The corresponding grayscale images are shown in Figure S7; (b) Interactive plot of the portion of the FP to show the distribution of sweat pores on the ridges of the FP; (c) Fluctuations in the gray value over the FP indicated by the white bar in the panel "a" (Scale bar: $5 \mathrm{~mm}$ ). 

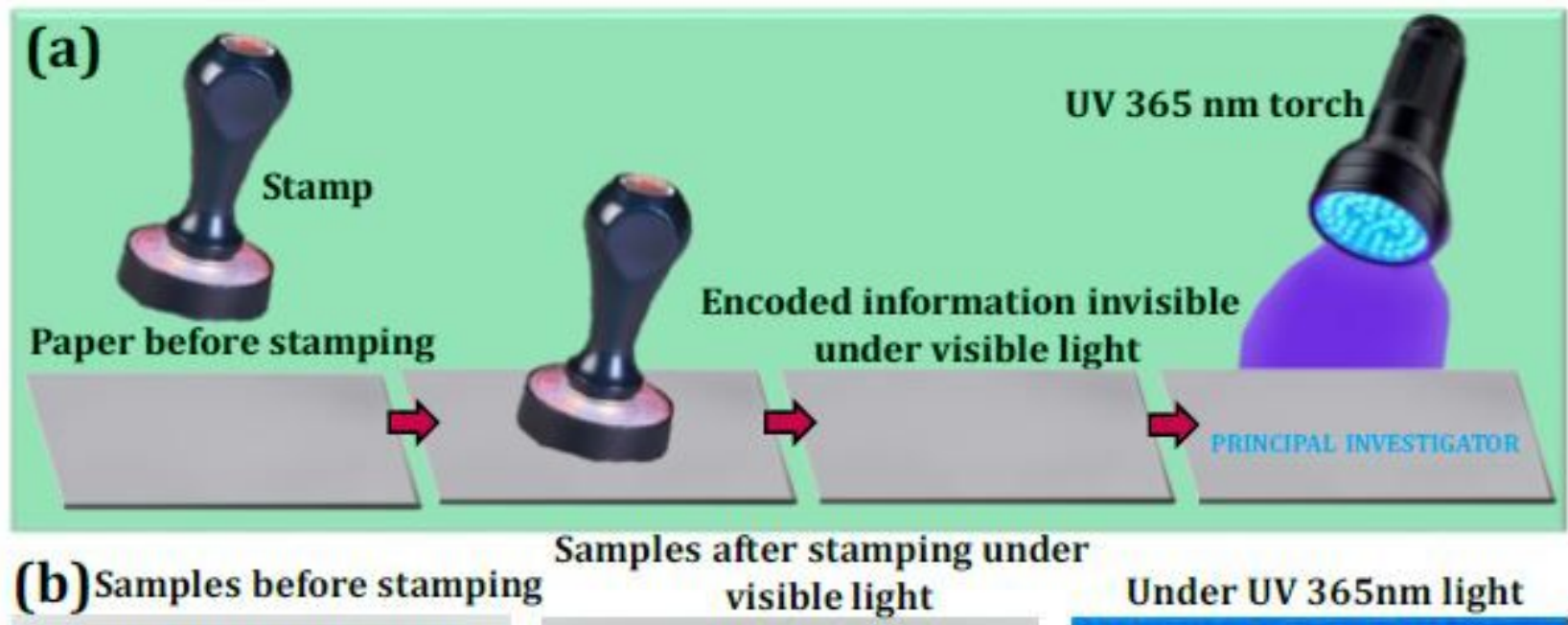

\section{Samples after stamping under}

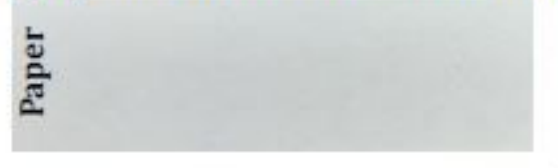
visible light
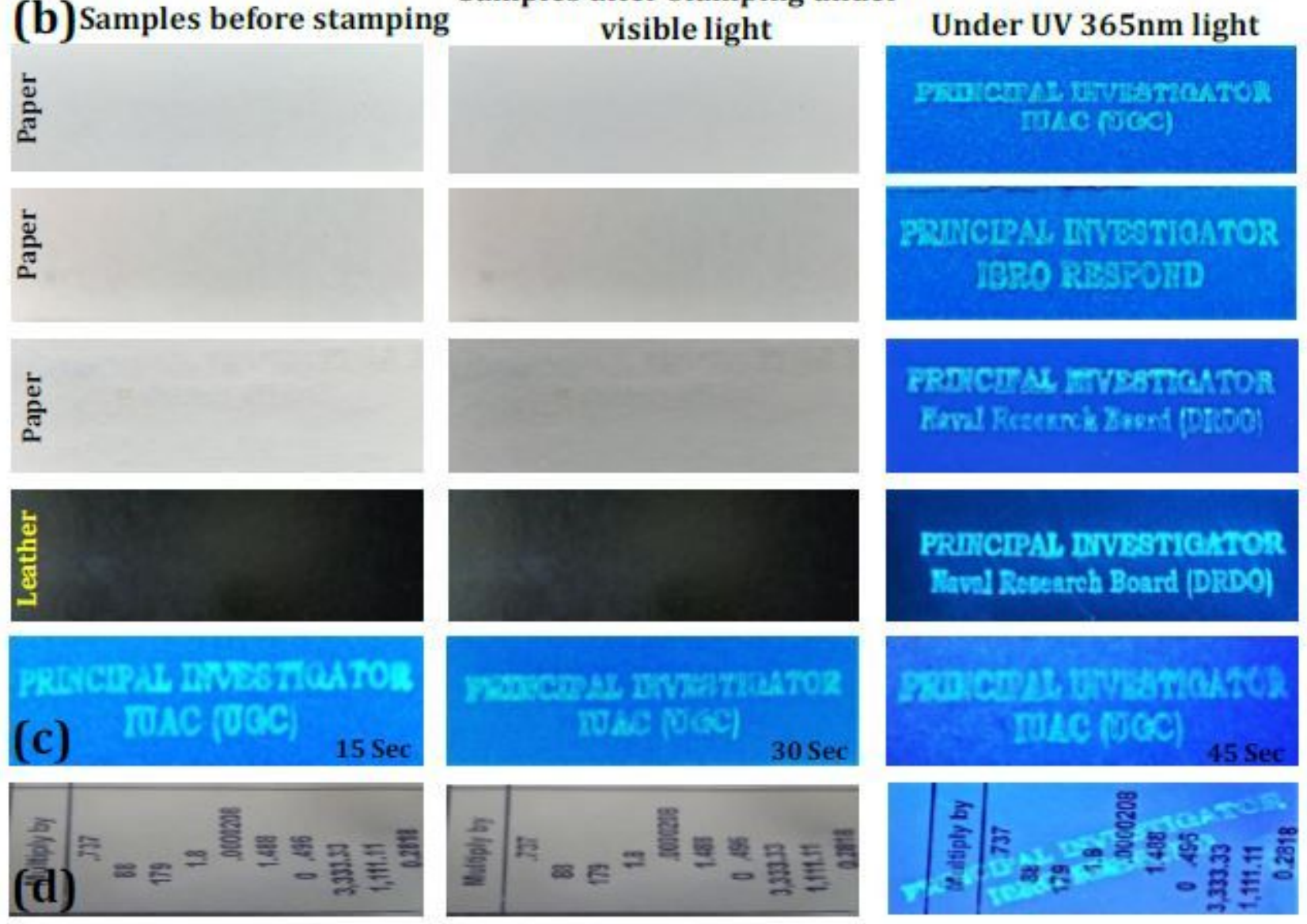

\section{Figure 9}

(a) Schematic illustration of different input anti-counterfeiting patterns developed on paper surface by following stamping method. UV $365 \mathrm{~nm}$ light is used as an excitation source to decode the patterns. The developed information was in situ photographed using a DSLR Canon EOS 100D camera; (b) Photographs of the word "PRINCIPAL INVESTIGATOR IUAC (UGC)", "PRINCIPAL INVESTIGATOR ISRO RESPOND" and "PRINCIPAL INVESTIGATOR Naval Research Board (DRDO)" stamped by using FDIP solution on paper and leather surfaces. Left panels are plane surfaces without any stamping under normal light. Middle panels of each photographs exhibit images in visible light after stamping. Right panels are corresponding photographic images of input patterns under UV $365 \mathrm{~nm}$ light; (c) Fluorescence 
images on paper developed with various stamping time under UV $365 \mathrm{~nm}$ light irradiation; (d) Images of anti-counterfeiting water-mark were developed using FDIP solution on the complex paper surface by stamped method.
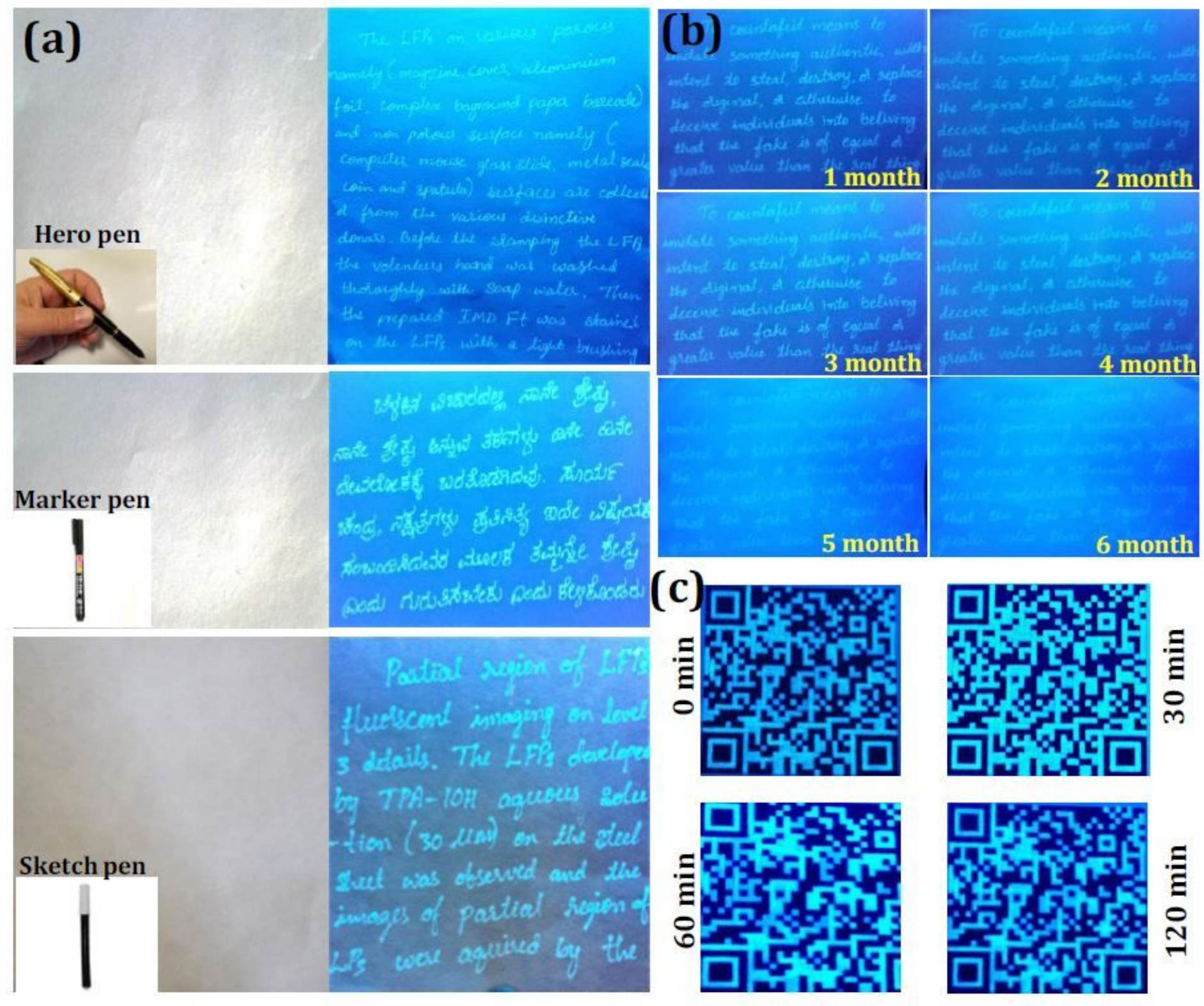

Figure 10

(a) Photographic images of English and Kannada words handwritten by hero pen, marker pen and sketch pen under visible and UV $365 \mathrm{~nm}$ light excitation; (b) Fluorescent images of decoded anti-counterfeiting information stored in different time periods at RT; (c) Photostability of developed QR code information treated with different UV irradiation time periods. 


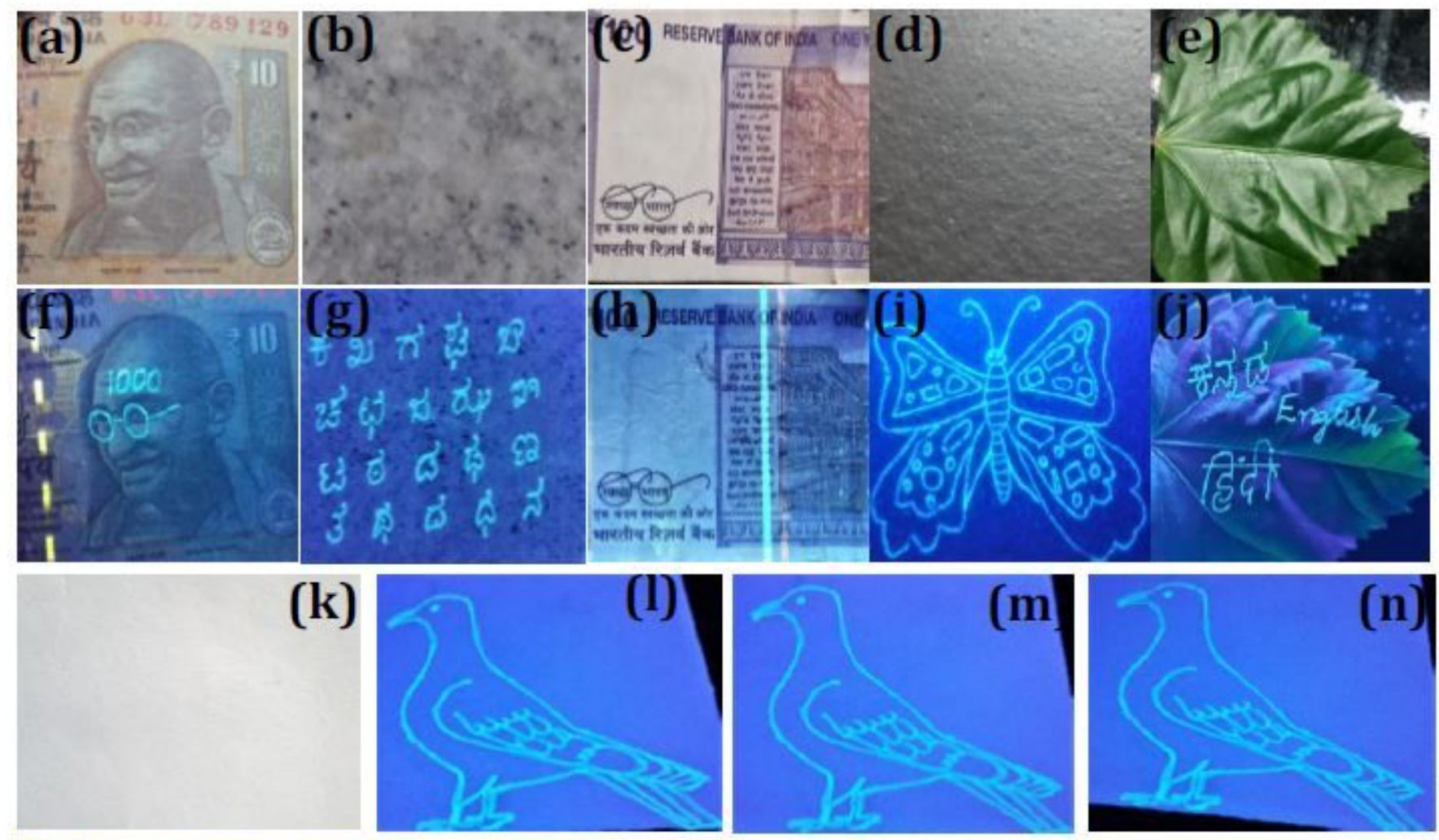

\section{(0)}

(p)
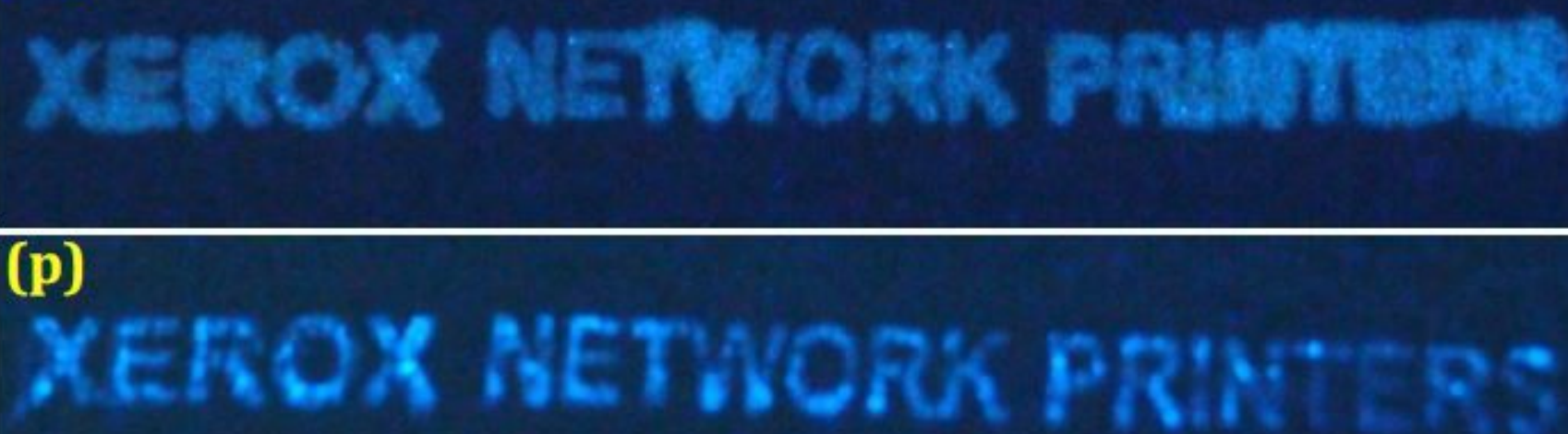

\section{(q)}

\section{Figure 11}

Images of encoded information on various surfaces under (a-e) normal light, (f-j) UV 365 nm light excitation; (k-n) Photographic images of encoded handwritten information on the glassy ceramic tile surface and treated with various time period and temperature ( 30 days at RT, below $90{ }^{\circ} \mathrm{C}$ for $24 \mathrm{hrs}, 100$ ${ }^{\circ} \mathrm{C}$ for $30 \mathrm{~min}$ ); fluorescent images of decoded patterns under UV $365 \mathrm{~nm}$ light excitation showing high reliability on the paper surface by treated with (o) water, (p) oil and (q) acetone for $\sim 12 \mathrm{~h}$. 


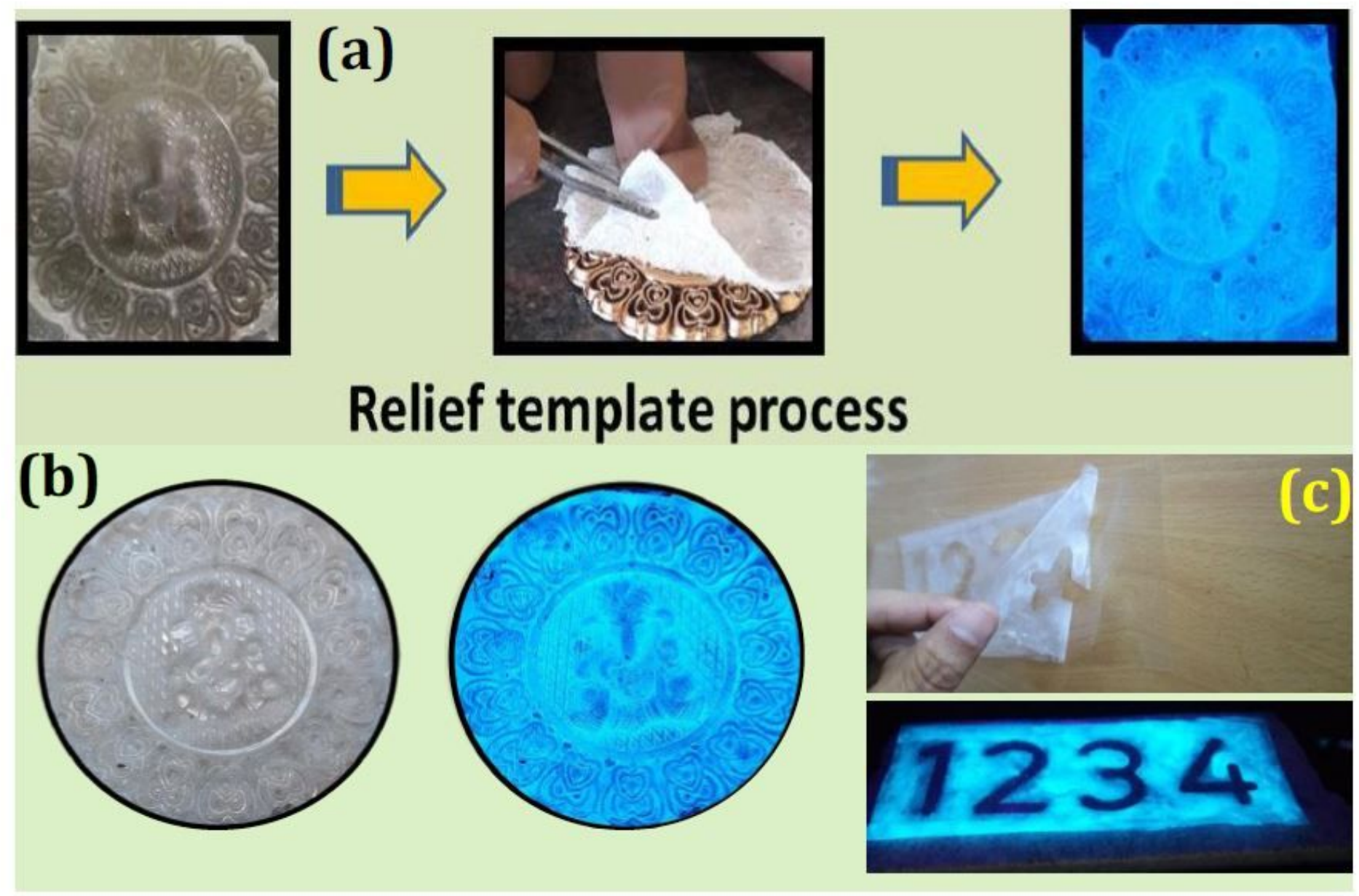

Figure 12

(a) Schematic representation of simple ink-free relief template method; (b) Photographed images of developed "lord Ganesha" film by relief template method upon visible and UV $365 \mathrm{~nm}$ irradiation; (c) Xylographic images developed by following ink-free intaglio printing method with prepared PVA/FDIP under normal and UV $365 \mathrm{~nm}$ light.

\section{Supplementary Files}

This is a list of supplementary files associated with this preprint. Click to download.

- Supplimentorylnformation.docx 